Conflict of interest: S.C. de Cassan, M.K. Higgins, A.V.S. Hill, and S.J. Draper are named inventors on patent applications (patent nos. CB1413530.5, CB1016471.3, and W0/2008/122811) covering malaria vaccines and immunization regimens. A. Nicosia was an employee of and shareholder in Okairòs (since acquired by ClaxoSmithKline), which is developing vectored vaccines for a number of diseases. T. Jørgensen and W.A. de Jongh are employees of, and W.A. de Jongh is a shareholder in, ExpreS2ion Biotechnologies, which has developed and is marketing the ExpreS ${ }^{2}$ cell expression platform. C.E. Chitnis is a named inventor on a patent covering PvDBP_RII (patent no. Wo/1996/040766).

License: This work is licensed under the Creative Commons Attribution 4.0 International License. To view a copy of this license, visit http:// creativecommons.org/licenses/by/4.0/

Submitted: February 28, 2017

Accepted: May 16, 2017

Published: June 15, 2017

Reference information: JCI Insight. 2017;2(12):e93683. https://doi.org/10.1172/jci. insight.93683.

\section{Human vaccination against Plasmodium vivax Duffy-binding protein induces strain-transcending antibodies}

\author{
Ruth O. Payne, ${ }^{1}$ Sarah E. Silk, ${ }^{1}$ Sean C. Elias, ${ }^{1}$ Kathryn H. Milne, ${ }^{1}$ Thomas A. Rawlinson, \\ David Llewellyn, ${ }^{1}$ A. Rushdi Shakri, ${ }^{2}$ Jing Jin, ${ }^{1}$ Geneviève M. Labbé, ${ }^{1}$ Nick J. Edwards, ${ }^{1}$ \\ Ian D. Poulton, ${ }^{1}$ Rachel Roberts, ${ }^{1}$ Ryan Farid, ${ }^{3}$ Thomas Jørgensen, ${ }^{4}$ Daniel C.W. Alanine, ${ }^{1}$ \\ Simone C. de Cassan, ${ }^{1}$ Matthew K. Higgins, ${ }^{5}$ Thomas D. Otto, ${ }^{6}$ James S. McCarthy, ${ }^{3}$ \\ Willem A. de Jongh, ${ }^{4}$ Alfredo Nicosia, ${ }^{7,89}$ Sarah Moyle, ${ }^{10}$ Adrian V.S. Hill, ${ }^{1}$ Eleanor Berrie, ${ }^{10}$ \\ Chetan E. Chitnis, ${ }^{2,11}$ Alison M. Lawrie, ${ }^{1}$ and Simon J. Draper ${ }^{1}$
}

'The Jenner Institute, University of Oxford, Oxford, United Kingdom. 'International Center for Cenetic Engineering and Biotechnology, Aruna Asaf Ali Marg, New Delhi, India. ${ }^{3}$ IMR Berghofer Medical Research Institute, Herston, Queensland, Australia. ${ }^{4}$ ExpreSion Biotechnologies, SCION-DTU Science Park, Hørsholm, Denmark. ${ }^{5}$ Department of Biochemistry, University of Oxford, Oxford, United Kingdom. ${ }^{6}$ Wellcome Trust Sanger Institute, Cambridge, United Kingdom. ${ }^{7}$ ReiThera SRL (formerly Okairòs SRL), Viale Città d'Europa, Rome, Italy. ${ }^{8} \mathrm{CEINGE,} \mathrm{Naples,} \mathrm{Italy.}{ }^{9}$ Department of Molecular Medicine and Medical Biotechnology, University of Naples Federico II, Naples, Italy. ${ }^{10} \mathrm{Clinical}$ Biomanufacturing Facility, University of Oxford, Oxford, United Kingdom. ${ }^{11}$ Institut Pasteur, Department of Parasites and Insect Vectors, Paris, France.

BACKCROUND. Plasmodium vivax is the most widespread human malaria geographically; however, no effective vaccine exists. Red blood cell invasion by the $P$. vivax merozoite depends on an interaction between the Duffy antigen receptor for chemokines (DARC) and region II of the parasite's Duffy-binding protein (PvDBP_RII). Naturally acquired binding-inhibitory antibodies against this interaction associate with clinical immunity, but it is unknown whether these responses can be induced by human vaccination.

METHODS. Safety and immunogenicity of replication-deficient chimpanzee adenovirus serotype 63 (ChAd63) and modified vaccinia virus Ankara (MVA) viral vectored vaccines targeting PvDBP_RII (Salvador I strain) were assessed in an open-label dose-escalation phase la study in 24 healthy UK adults. Vaccines were delivered by the intramuscular route in a ChAd63-MVA heterologous primeboost regimen using an 8 -week interval.

RESULTS. Both vaccines were well tolerated and demonstrated a favorable safety profile in malaria-naive adults. PvDBP_RII-specific ex-vivo IFN- $\gamma$ T cell, antibody-secreting cell, memory B cell, and serum IgG responses were observed after the MVA boost immunization. Vaccine-induced antibodies inhibited the binding of vaccine homologous and heterologous variants of recombinant PvDBP_RII to the DARC receptor, with median 50\% binding-inhibition titers greater than 1:100.

CONCLUSION. We have demonstrated for the first time to our knowledge that strain-transcending antibodies can be induced against the PvDBP_RII antigen by vaccination in humans. These vaccine candidates warrant further clinical evaluation of efficacy against the blood-stage $P$. vivax parasite.

TRIAL REGISTRATION. Clinicaltrials.gov NCT01816113.

FUNDING. Support was provided by the UK Medical Research Council, UK National Institute of Health Research Oxford Biomedical Research Centre, and the Wellcome Trust.

\section{Introduction}

Five species of Plasmodium parasite are known to cause malaria following human infection, with $P$. falciparum the major causative agent of deaths in sub-Saharan Africa and thus historically the dominant focus of vaccine development efforts (1). However, a second parasite species, $P$. vivax, is more widespread geographically and also constitutes a significant proportion of human malaria cases. Indeed, recent data suggest 2.5 
billion people are living at risk of $P$. vivax infection in the Americas, Central and Southeast Asia (2), as well as Africa (3), highlighting significant levels of morbidity that have been chronically underappreciated (4). Consequently, the revised Malaria Vaccine Technology Roadmap to 2030 (5) now recognizes the importance of $P$. vivax and calls for a vaccine to achieve $75 \%$ efficacy over 2 years - equally weighted with $P$. falciparum in an era of renewed political will to move towards malaria elimination and eradication.

Different vaccine strategies target different stages of the malaria parasite's complex life cycle. To date, 2 subunit vaccines targeting the pre-erythrocytic stage $P$. vivax circumsporozoite protein (PvCSP), based on recombinant protein- or long synthetic peptide-in-adjuvant formulations, have reached clinical trials $(6,7)$. The soluble recombinant protein candidate, VMP001, delivered in GlaxoSmithKline's (GSK's) proprietary Adjuvant System AS01B, showed robust immunogenicity in healthy US volunteers but failed to induce sterile protection following $P$. vivax controlled human malaria infection (CHMI) using a mosquito bite protocol; however, a small but significant delay in time to parasitemia was seen in 16 of 27 vaccinated subjects compared with the control group (7). A virus-like particle (VLP) using the same antigen fused to hepatitis B surface antigen (HBsAg), expressed in Saccharomyces cerevisiae and designated CSV-S,S, showed modest improvements in immunogenicity when tested in rhesus macaques with AS01 (8), but has not progressed to clinical testing. In 2 other phase Ia clinical trials, a soluble recombinant protein vaccine targeting the sexual-stage ookinete surface protein Pvs 25 was tested in 2 different adjuvants. This vaccine candidate, called Pvs25H, showed transmission-blocking activity in a direct membrane feeding assay when formulated with Alhydrogel (9), but vaccinations with Montanide ISA 51 were halted due to unexpected reactogenicity (10). None of these preerythrocytic or transmission-blocking candidate subunit vaccines remain in active clinical development.

Vaccines targeting the asexual blood-stage infection form an alternative and complementary approach to vaccines against the other life cycle stages, seeking to control and clear parasitemia in order to prevent clinical disease and death as well as onward transmission. Although many candidates have been assessed over the years for $P$. falciparum (1), no clinical trials of vaccines against merozoite ligands involved in erythrocyte invasion have been reported for $P$. vivax (11). The Duffy-binding proteins (DBPs), or erythrocyte-binding ligands/antigens (EBL/EBA), are a family of micronemal parasite proteins that are functionally conserved across Plasmodium species. All parasites have at least one EBL, and in many cases these lead to redundancy, as has been well established in $P$. falciparum (12). However in the case of $P$. vivax, invasion of host red blood cells $(\mathrm{RBC})$ is restricted to $\mathrm{CD} 71^{+}$reticulocytes $(13)$ and believed to necessitate the interaction of the $P$. vivax Duffy-binding protein (PvDBP) with the human Duffy antigen receptor for chemokines (DARC/Fy) (14). Notably, Duffy-negative individuals are protected from blood-stage $P$. vivax infection, an observation first reported by Miller et al. in 1976 (15), confirmed by CHMI studies (16), and associated geographically with low-level endemicity in sub-Saharan Africa (3). Consistent with this, genetic knockout of the orthologous simian malaria P. knowlesi $\mathrm{DBP} \alpha$ gene also prevents invasion of Duffy-positive erythrocytes in vitro (17). However, this paradigm of an essential RBC invasion pathway has been challenged in recent years with reports of $P$. vivax infection in Duffy-negative individuals $(3,18)$ and a growing appreciation of the complexity of other families of invasion ligands, such as the reticulocyte-binding proteins (PvRBPs) (19). In parallel, a PvDBP gene duplication in $P$. vivax isolates (20) has also been reported, likely representing a second erythrocyte-binding protein (EBP2) (21), although studies have not linked this gene to Duffy-negative infection $(22,23)$. Therefore, although the complete molecular basis of $P$. vivax invasion into DARC-negative erythrocytes remains unknown, it may still involve PvDBP.

In the case of PvDBP, a conserved, extracellular, cysteine-rich region known as region II (PvDBP RII) contains the receptor-binding domain of PvDBP. Structural analyses of this domain have shown that PvDBP_RII dimers bind either 1 or 2 DARC ectodomains, creating distinct heterotrimeric and heterotetrameric architectures $(24,25)$. Immunization of mice, rabbits, and nonhuman primates (NHPs) using PvDBP RII-based vaccines induces binding-inhibitory antibodies (BIAbs) (26-28), and those raised against the $P$. knowlesi $\mathrm{DBP} \alpha$ ortholog can block RBC invasion by this parasite in vitro (29). In humans, naturally acquired high-titer BIAbs against PvDBP_RII have been associated with reduced risk of $P$. vivax infection, lower $P$. vivax parasite densities following infection, and decreased risk of clinical malaria (30,31). Consequently, PvDBP_RII remains the most promising subunit vaccine target against $P$. vivax merozoites; however, this antigen has never progressed to clinical trials and no data are available on the ability of vaccines to induce effective immune responses in humans.

With regard to antibody induction by vaccination, the mainstay approach has been the development of recombinant protein- or VLP-in-adjuvant formulations. An alternative strategy has used recombinant viral 
vectored vaccines to deliver protein antigens of interest with the key aim of inducing antibodies in conjunction with $\mathrm{T}$ cell responses. The most successful approach to date has utilized a recombinant replication-deficient adenovirus (of human or simian serotype) to prime the immune response, followed by a booster vaccination (typically 8 weeks later) with an attenuated poxvirus recombinant for the same antigen (32). These vectors have shown high-titer antibody induction against numerous difficult-to-express malaria antigens in animal models, including NHPs $(33,34)$. We, and others, have previously reported such viral vectored vaccines to be safe and immunogenic for $\mathrm{T}$ cells and antibodies in healthy adult UK and US volunteers when delivering numerous $P$. falciparum antigens, including the pre-erythrocytic antigen multiple-epitope string fused to thrombospondinrelated adhesion protein (ME-TRAP) (35) and circumsporozoite protein (PfCSP) (36), as well as the blood-stage antigens merozoite surface protein 1 (PfMSP1) (37) and apical membrane antigen 1 (PfAMA1) (38, 39). In 2014 and 2015, the same adenovirus-poxvirus vectored vaccine technologies were developed rapidly for Ebola (40).

Here, we report the safety and immunogenicity of a similar approach in an open-label dose-escalation phase Ia study in healthy UK adults using replication-deficient chimpanzee adenovirus serotype 63 (ChAd63) and the attenuated orthopoxvirus modified vaccinia virus Ankara (MVA) encoding PvDBP_RII from the Salvador I (SalI) reference strain of $P$. vivax. These vaccines have been previously shown to be immunogenic in mice and rabbits (27). Now we show that these vaccines demonstrate a favorable safety profile in malarianaive adults, and confirm to our knowledge for the first time that substantial PvDBP_RII-specific antibodies and $\mathrm{B}$ cell and $\mathrm{T}$ cell responses can be induced by immunization in humans. Vaccine-induced serum antibodies were capable of inhibiting the in vitro binding of vaccine homologous and heterologous variants of recombinant PvDBP_RII to the DARC receptor.

\section{Results}

Twenty-four healthy adult volunteers were enrolled into the VAC051 trial to test the ChAd63-MVA PvDBP_RII vaccine in an open-label, dose-escalation study design. Thirty UK adult volunteers were screened in total, of which 24 were enrolled (Figure 1). Four volunteers were recruited to groups 1 and 2A, and 8 volunteers to groups 2B and 2C. In total, 15 females and 9 males were enrolled. The mean age of volunteers was 25 years 9 months (range 18-40 years). Four volunteers were enrolled into group 1 and received $5 \times 10^{9}$ viral particles (vp) of the ChAd63 PvDBP_RII vaccine. Following a safety review, the dose of ChAd63 PvDBP_RII was increased to $5 \times 10^{10} \mathrm{vp}$ for group 2. Four volunteers in group 2A received ChAd63 PvDBP_RII alone, while volunteers in groups $2 \mathrm{~B}$ and $2 \mathrm{C}$ received ChAd63 PvDBP_RII followed 8 weeks later with a boost vaccination of MVA PvDBP_RII at a dose of $1 \times 10^{8}$ plaque-forming units (PFU) or $2 \times 10^{8} \mathrm{PFU}$, respectively. One volunteer withdrew from group 2B prior to the MVA PvDBP_RII vaccination due to personal commitments and was not replaced, resulting in 23 volunteers completing follow-up as per protocol.

ChAd63 and MVA PvDBP_RII show a favorable safety profile in healthy UK adult volunteers. There were no serious adverse events (AEs) or unexpected reactions during the course of the trial and no volunteers withdrew due to vaccine-related AEs. ChAd63 PvDBP_RII and MVA PvDBP_RII demonstrated favorable safety profiles, similar to those seen in previous clinical trials with the same viral vectors recombinant for $P$. falciparum malaria antigens (35-38). All AEs following ChAd63 PvDBP_RII $5 \times 10^{9}$ vp were mild, as were the vast majority in group 2, although some volunteers did report moderate or severe AEs following immunization with the full dose. The higher dose of MVA PvDBP_RII was more reactogenic than the lower dose, with half of the volunteers reporting at least 1 severe AE, although no systemic AE was reported as severe for more than 24 hours. The maximum severities of solicited local and systemic AEs reported by volunteers following each vaccination are shown in Figure 2. All unsolicited AEs considered possibly, probably, or definitely related to either vaccination were mild in nature (Supplemental Table 1; supplemental material available online with this article; https://doi.org/10.1172/jci.insight.93683DS1). There was only 1 laboratory AE following ChAd63 PvDBP_RII that was considered possibly, probably, or definitely related to vaccination: a mild lymphopenia in 1 volunteer vaccinated with $5 \times 10^{10} \mathrm{vp}$. Similarly, there was only 1 laboratory AE following MVA PvDBP_RII that was considered possibly, probably, or definitely related to vaccination: a moderate eosinophilia in 1 volunteer vaccinated with $1 \times 10^{8} \mathrm{PFU}$, which peaked more than 4 weeks after vaccination. Both of these laboratory AEs resolved spontaneously.

ChAd63 and MVA PvDBP_RII expand IFN- $\gamma$ T cell responses in healthy UK adult volunteers. The kinetics and magnitude of the PvDBP-specific T cell response were assessed over time by ex vivo IFN- $\gamma$ ELISPOT following restimulation of peripheral blood mononuclear cells (PBMCs) with 20-mer peptides overlapping by 10 amino acids (aa) spanning the entire PvDBP_RII insert present in the vaccines (Figure 3). Vaccination 


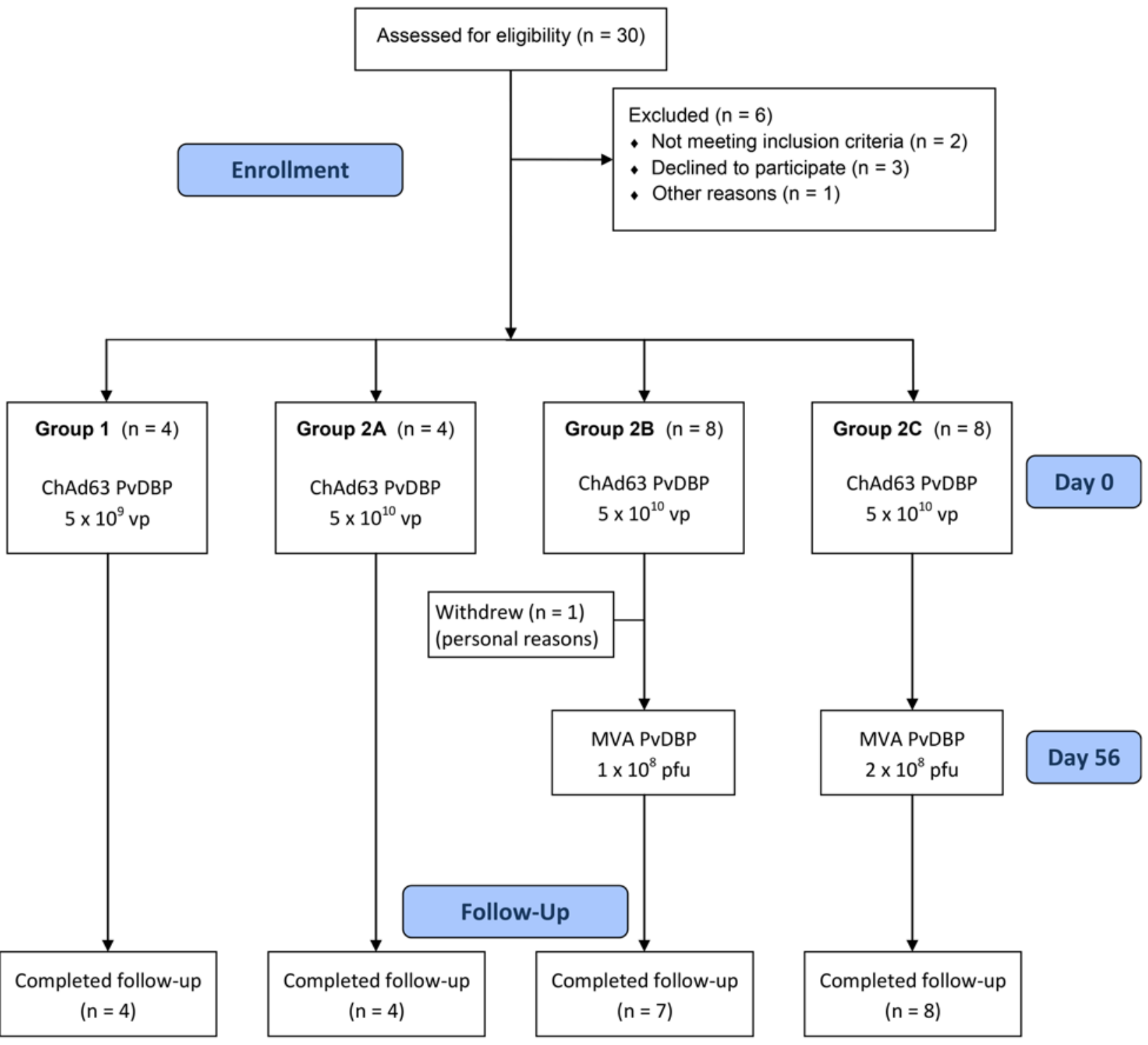

Figure 1. VAC051 flow chart of study design and volunteer recruitment. Recruitment for the VAC051 study took place between May 2013 and February 2014. The final follow-up visit took place in July 2014. All immunizations were administered intramuscularly, with sequential vaccines administered into the deltoid of alternating arms.

with ChAd63-MVA PvDBP_RII induced antigen-specific T cell responses in all volunteers, with individual responses shown in Supplemental Figure 1 and median responses to the total vaccine insert shown for each group in Figure 3A. Following ChAd63 PvDBP_RII prime, there was no significant difference between median responses in the lower-dose group 1 in comparison with group 2 at the peak of the response on day 14 (median 787 [range 140-1,893] vs. 937 [range 96-4,141] spot-forming units [SFU]/million PBMCs in groups 1 versus 2 , respectively; $n=4$ vs. $20, P=0.79$ by Mann-Whitney test) (Figure 3B). Responses subsequently followed classical $\mathrm{T}$ cell kinetics and contracted by day 56 (Figure 3A). Administration of MVA PvDBP_RII significantly boosted these responses in all volunteers as measured 1 week later on day 63 (groups $2 \mathrm{~B}$ and $2 \mathrm{C}$ vs. 2A, Kruskal-Wallis test with Dunn's multiple comparison test) (Figure 3C), reaching medians of 2,061 (range 1,232-2,844) and 2,459 (range 675-4,336) SFU/million PBMCs in groups 2B and 2C, respectively, versus 368 (range 127-699) SFU/million PBMCs in group 2A. However, there was no significant difference between the 2 groups who received the different doses of MVA PvDBP_RII $(P=0.96$, Mann-Whitney test). T cell responses were spread across the whole PvDBP_RII antigen, with responses 
A

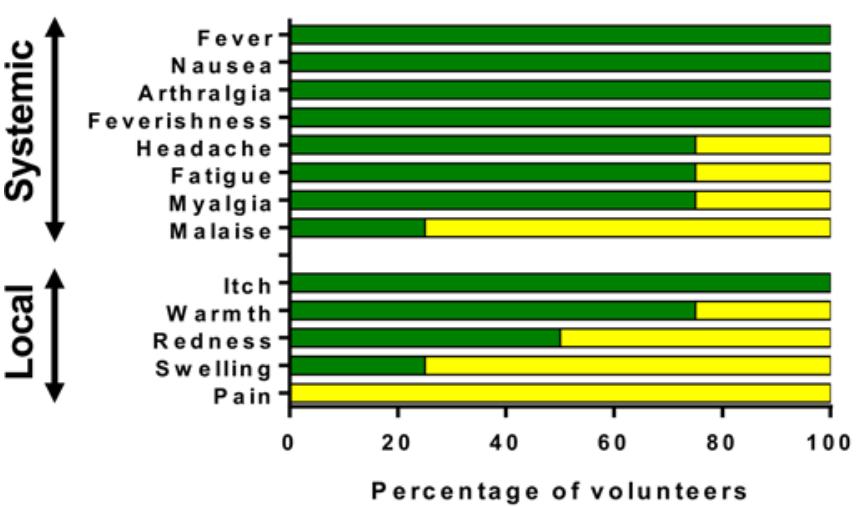

C

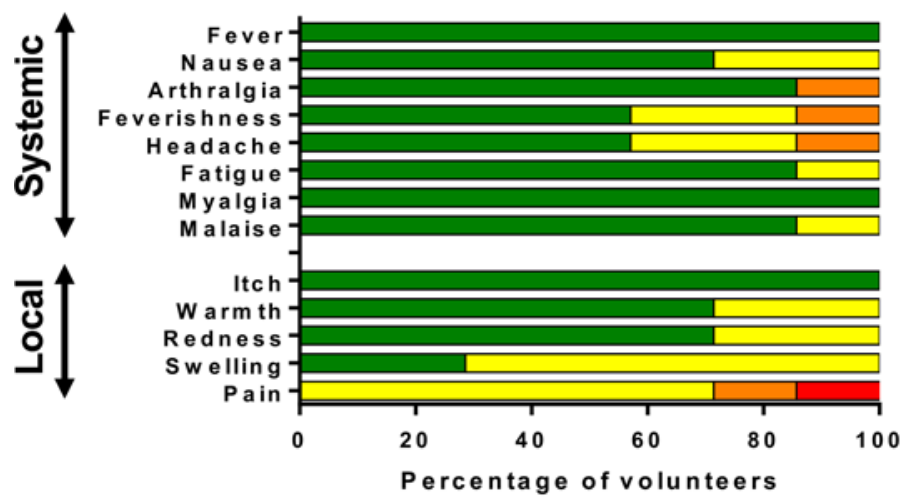

None

Group 1 post-ChAd63

Group 2B post-MVA

Mild
B

Group 2 post-ChAd 63

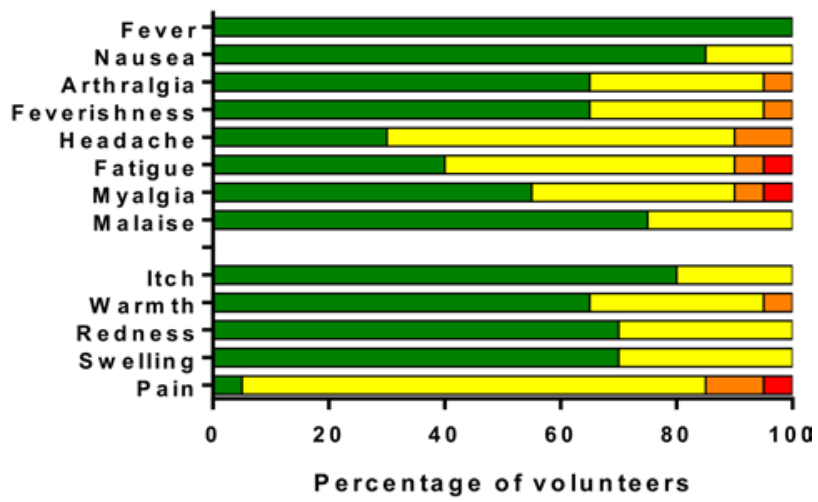

D

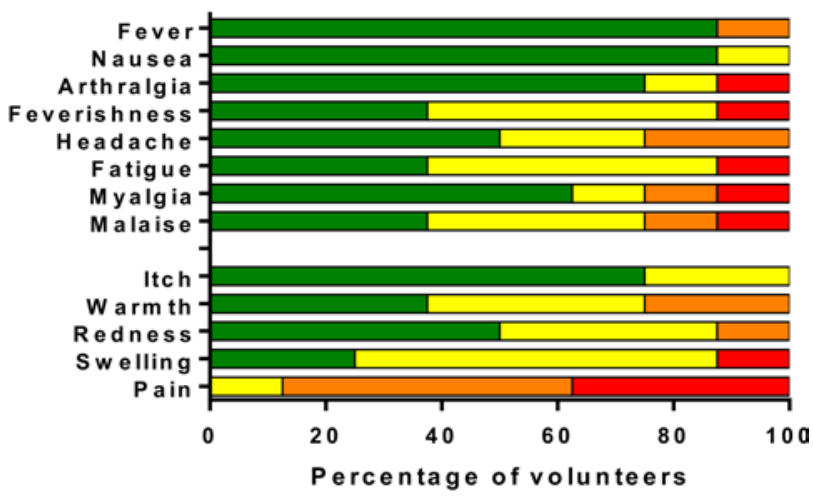

Moderate

\section{Severe}

Figure 2. Solicited AEs following vaccination with ChAd63 and MVA PvDBP_RII. The solicited local and systemic adverse events (AEs) recorded for 14 days following ChAd63 PvDBP_RII and for 7 days following MVA PvDBP_RII are shown at the maximum severity reported by all volunteers. (A) Four volunteers received $5 \times 10^{9}$ viral particles (vp) ChAd63 PvDBP_RII (group 1), and (B) 20 received $5 \times 10^{10}$ vp (group 2). (C) Seven of the group 2 volunteers went on to receive MVA PvDBP_RII $1 \times 10^{8} \mathrm{PFU}$ (group $2 \mathrm{~B}$ ), and (D) 8 received $2 \times 10^{8} \mathrm{PFU}$ (group $2 \mathrm{C}$ ). ChAd63, replication-deficient chimpanzee adenovirus serotype 63; MVA, modified vaccinia virus Ankara; PvDBP_RII, region II of the $P$. vivax Duffy-binding protein.

detected in all 6 of the peptide pools used in the ELISPOT assay (Supplemental Figure 2). Following the peak at day 63 , responses contracted but were maintained above baseline at the end of the study period, with significantly better maintained responses at day 140 in group $2 \mathrm{C}$ as compared with group 2B (median 1,871 vs. $385 \mathrm{SFU} /$ million PBMCs) $(P=0.03$, Mann-Whitney test) (Figure 3D).

ChAd63 and MVA PvDBP_RII induce serum antibody responses and memory $B$ cells in healthy UK adult volunteers. The kinetics and magnitude of the anti-PvDBP_RII serum IgG antibody response were assessed over time by ELISA against recombinant protein (Figure 4). Priming vaccination with $5 \times 10^{10}$ vp ChAd63 PvDBP_RII followed by MVA PvDBP_RII boost induced antigen-specific IgG responses in all volunteers (groups $2 \mathrm{~B}$ and $2 \mathrm{C}$ ), with individual responses shown in Supplemental Figure 3 and median responses shown for each group in Figure 4A. Responses are reported in $\mu \mathrm{g} / \mathrm{ml}$ following conversion of ELISA arbitrary units (AU) by calibration-free concentration analysis (CFCA) (Supplemental Figure 4). Following ChAd63 PvDBP_RII prime with $5 \times 10^{9} \mathrm{vp}$, none of the 4 volunteers showed a detectable response on day 28 , in contrast to 12 of 20 volunteers who did show a response (median 0.3 , range $0-2.3 \mu \mathrm{g} / \mathrm{ml}$, $n=20)$ following priming with $5 \times 10^{10} \mathrm{vp}(P=0.07$, Mann-Whitney test) (Figure 4B). Responses were subsequently maintained in group 2 volunteers prior to administration of MVA PvDBP_RII, which led to a boost as measured 4 weeks later on day 84 (Figure $4 \mathrm{~A}$ ) - this reached significance for group $2 \mathrm{C}$ versus $2 \mathrm{~A}$ (Kruskal-Wallis test with Dunn's multiple comparison test) (Figure 4C). Responses in group 2C (median 15.6 , range $10.5-27.2 \mu \mathrm{g} / \mathrm{ml}, n=8$ ) were also modestly, but significantly, higher than in group 2B (median 
A

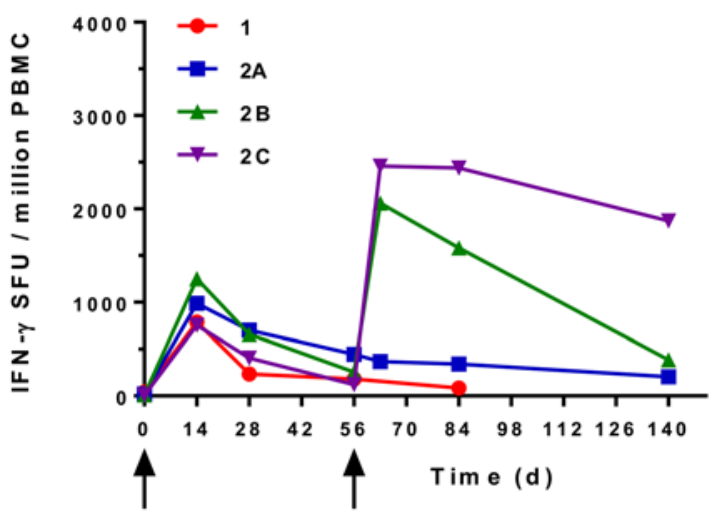

C

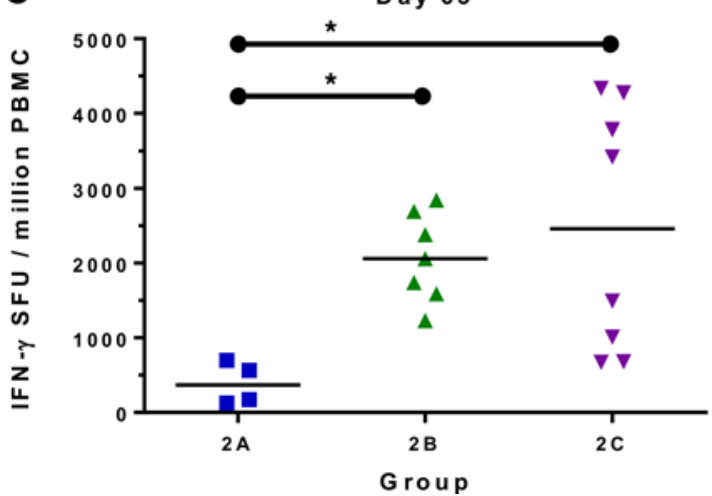

B

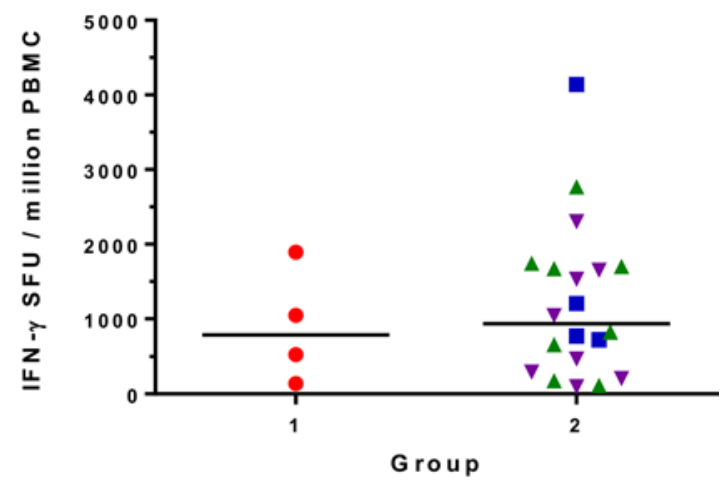

D

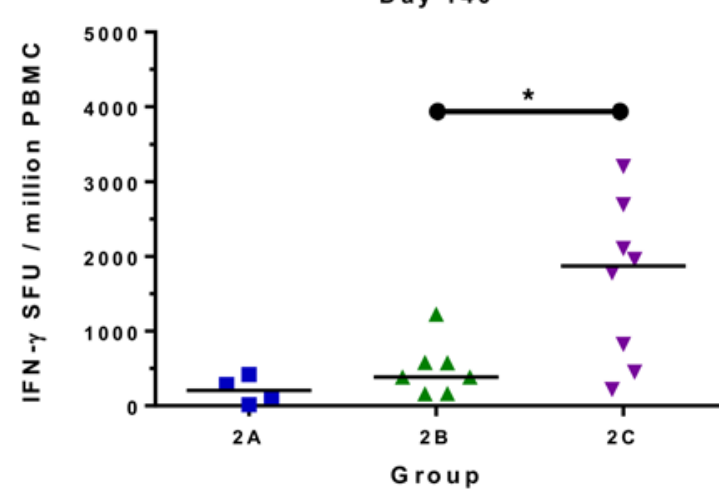

Figure 3. Ex-vivo IFN- $\gamma$ T cell response to vaccination. (A) Median ex vivo IFN- $\gamma$ ELISPOT responses in peripheral blood mononuclear cells (PBMCs) to the PVDBP_RII insert (summed response across all the individual peptide pools) shown for all groups. Individual responses are shown in Supplemental Figure 1. Median and individual responses are shown at (B) day 14, (C) day 63, and (D) day 140 . Symbols are coded according to group. ${ }^{*} P<0.05$. Responses between groups $1(n=4)$ and $2(n=20)$ at day 14 , and between groups $2 \mathrm{~B}(n=7)$ and $2 \mathrm{C}(n=8)$ at day 140 were assessed by Mann-Whitney test (B and D); responses between groups 2A $(n=4), 2 \mathrm{~B}(n=7)$, and 2C $(n=8)$ at day 63 were assessed by Kruskal-Wallis test with Dunn's multiple comparison test (C). SFU, spot-forming units; PvDBP_RII, region II of the $P$. vivax Duffy-binding protein.

8.8, range $5.5-23.7 \mu \mathrm{g} / \mathrm{ml}, n=8)$ at this peak time point $(P=0.014$, Mann-Whitney test). Serum antibody responses decreased by day 140 but were well maintained above preboost levels, with no significant difference between groups 2B and 2C ( $P=0.34$, Mann-Whitney test) (Figure 4D). Day 84 plasma were also tested against a panel of overlapping 20-mer linear peptides; however, few responses were detected above background, suggesting the vast majority of vaccine-induced anti-PvDBP_RII IgG recognize conformational, as opposed to linear, epitopes (Supplemental Figure 5).

The serum antibody response against PvDBP_RII as measured by ELISA at day 84 was composed of IgG1 and modest levels of IgG3 (Figure 4E), with little to no IgG2, IgG4, IgA, or IgM detectable above baseline (day 0) levels (Supplemental Figure 6). The avidity of the anti-PvDBP_RII IgG, as measured by a NaSCN-displacement ELISA, was similar at day 84 for all volunteers in groups $2 \mathrm{~B}$ and $2 \mathrm{C}$, with the $\mathrm{IC}_{50}$ ranging from 1.9 to $4.1 \mathrm{M}$. Avidity could only be measured for 1 vaccinee in group $2 \mathrm{~A}$ at this time point with an $\mathrm{IC}_{50}$ of $2.8 \mathrm{M}$, suggesting no change following MVA PvDBP_RII boost (Figure 4F).

Previous studies have shown that antibody-secreting cells (ASCs) can be detected in peripheral blood for a short time (around day 7) after MVA boost when using the ChAd63-MVA regimen (41, 42). PvDBP_ RII-specific ASC responses were assessed by ex-vivo ELISPOT using frozen PBMCs collected at the day 63 visit for volunteers in groups $2 \mathrm{~B}$ and 2C. Median responses of 49 versus 159 ASCs per million PBMCs were observed, respectively, but there was no significant difference between the 2 groups $(P=0.69$, MannWhitney test) (Figure 5A). ASC responses across both groups showed a trend to associate with peak serum antibody responses at day 84 , but this did not reach significance (Figure $5 \mathrm{~B}$ ).

Memory B cell ( $\mathrm{mBC}$ ) responses were also measured using an established cultured ELISPOT protocol, whereby mBCs within PBMCs undergo a 6-day polyclonal stimulation to form ASCs, which are then 
A

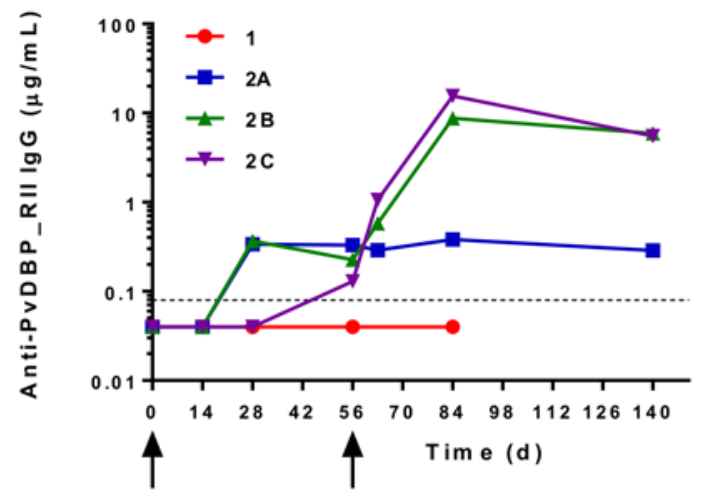

C

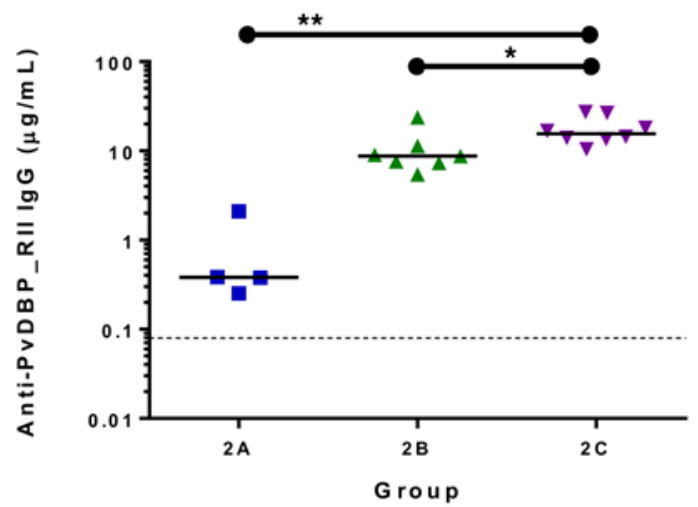

B

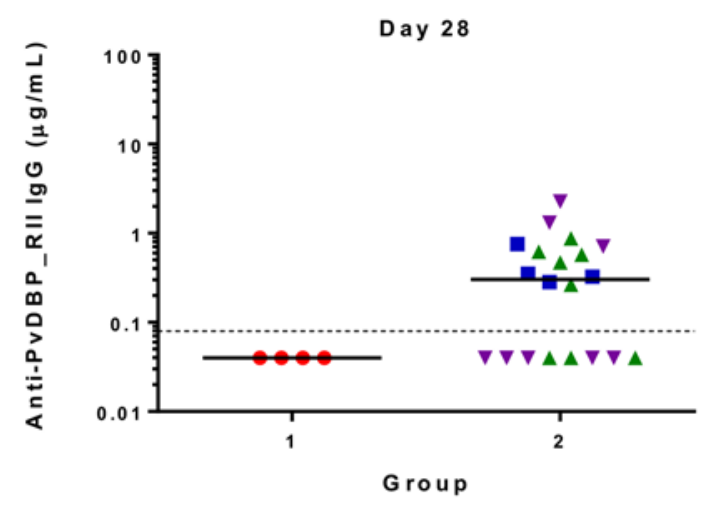

D

D ay 140

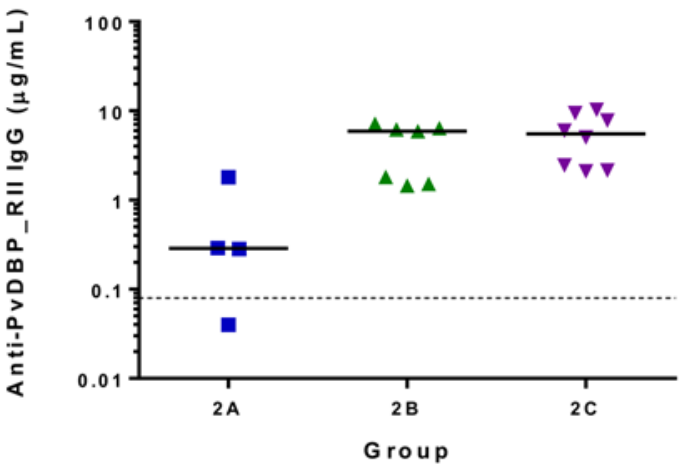

$\lg G 3$

$\mathbf{F}$
E

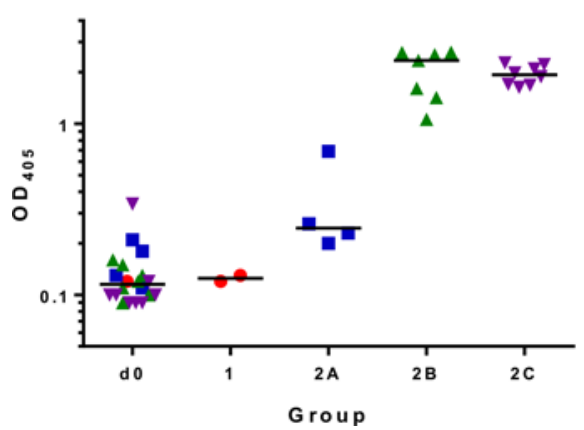

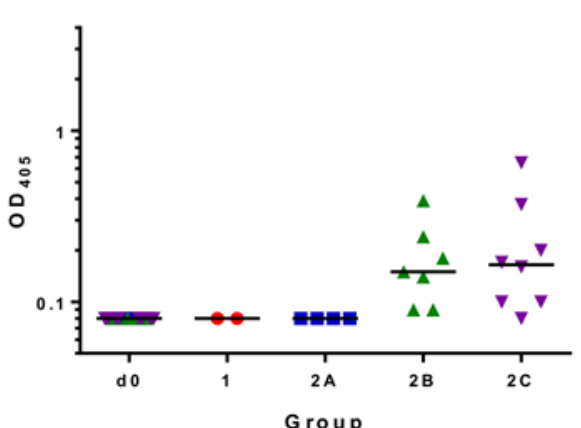

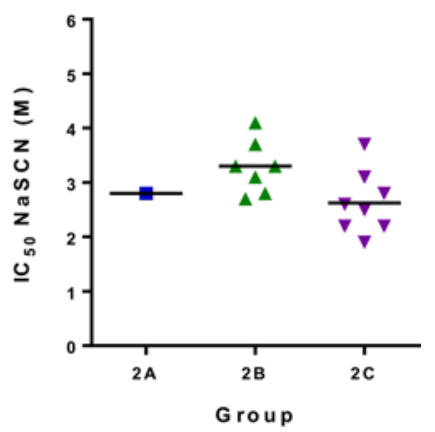

Figure 4. Serum antibody response to vaccination. (A) Median anti-PvDBP_RII serum total IgG responses shown for all groups over time. Individual responses are shown in Supplemental Figure 3. Median and individual responses are shown at (B) day 28, (C) day 84, and (D) day 140. The horizontal dotted line indicates the limit of detection of the assay. (E) Isotype profiles of serum antibody responses were assessed by ELISA. Responses are shown at baseline (d0) and for all groups at day 84. Individual and median responses are shown for IgG1 and IgG3; results for IgG2, IgG4, IgA, and IgM are shown in Supplemental Figure 6. (F) Avidity of serum IgG responses at day 84 was assessed by NaSCN-displacement PvDBP_RII ELISA and is reported as the molar (M) concentration of NaSCN required to reduce the starting OD in the ELISA by $50 \%\left(\mathrm{IC}_{50}\right)$. Symbols are coded according to group. ${ }^{*} P<0.05$, ${ }^{* *} P<0.01$. Responses in groups 2A ( $n=4), 2 \mathrm{~B}(n=7)$, and 2C $(n=8)$ were assessed by Kruskal-Wallis test with Dunn's multiple comparison test; responses between groups $2 \mathrm{~B}$ and $2 \mathrm{C}$ were assessed by Mann-Whitney test (C). PvDBP_RII, region II of the $P$. vivax Duffy-binding protein.

measured using the same protocol as for the ex vivo assay. These were measured for volunteers in groups $2 \mathrm{~B}$ and $2 \mathrm{C}$ at the day 84 time point (4 weeks after MVA boost) - most consistently identified as the peak of the $\mathrm{mBC}$ response in other trials of ChAd63-MVA $P$. falciparum blood-stage malaria vaccines $(41,42)$. Responses are reported as number of $\mathrm{mBC}$-derived PvDBP_RII-specific ASCs per million cultured PBMCs (Figure 5C), and as a percentage of total IgG-secreting ASCs (Figure 5D); in both cases these were significantly higher in group $2 \mathrm{C}$ than $2 \mathrm{~B}$ (Mann-Whitney test). These $\mathrm{mBC}$ responses across both groups also significantly correlated with peak serum antibody responses at day 84 (Figure 5, E and F). 
A

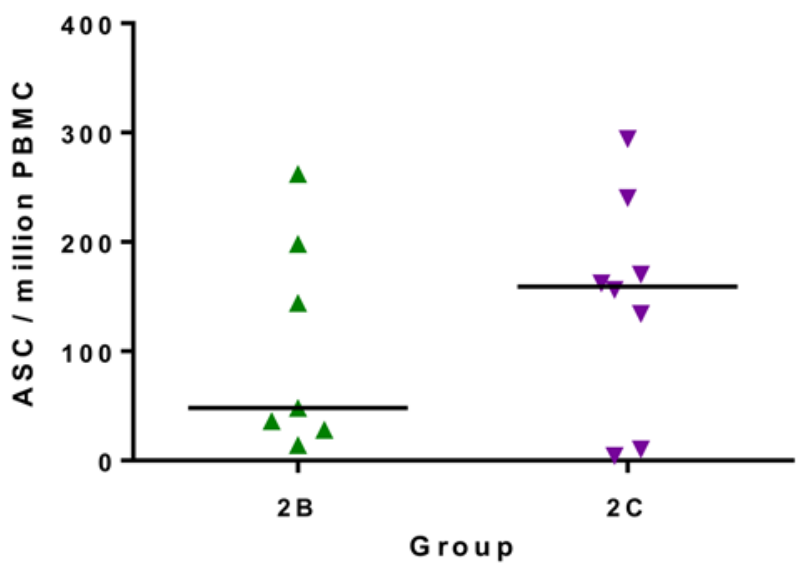

C

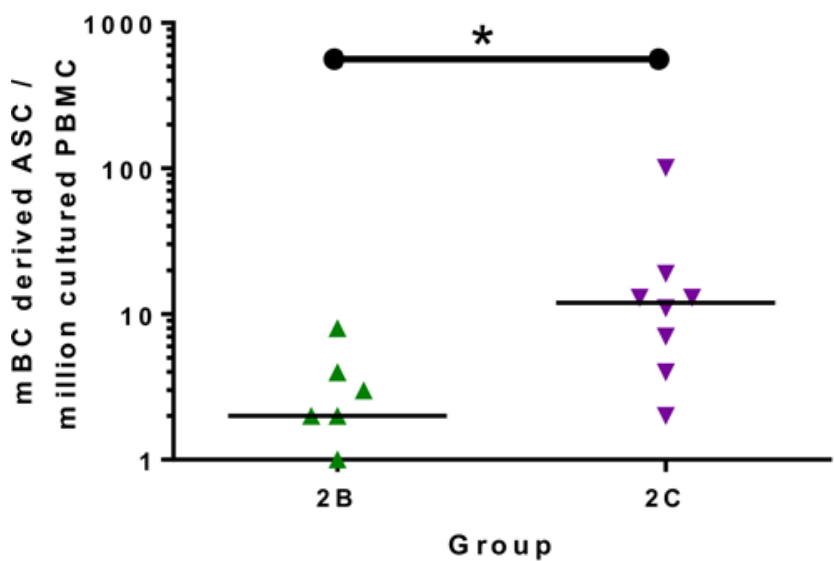

E

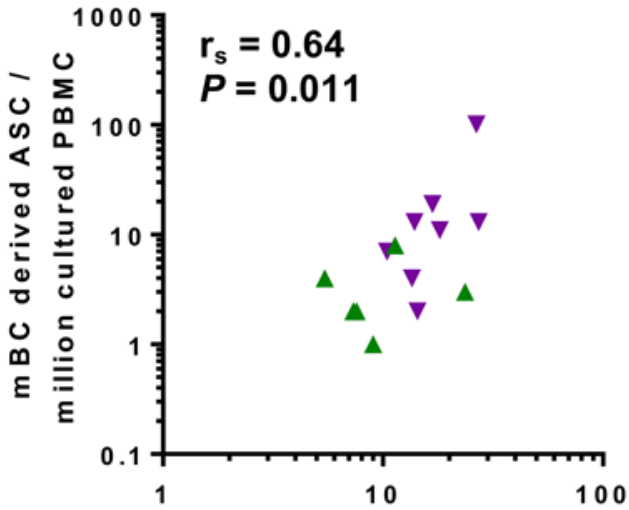

Anti-PvDBP_RII IgG $(\mu \mathrm{g} / \mathrm{mL})$
B

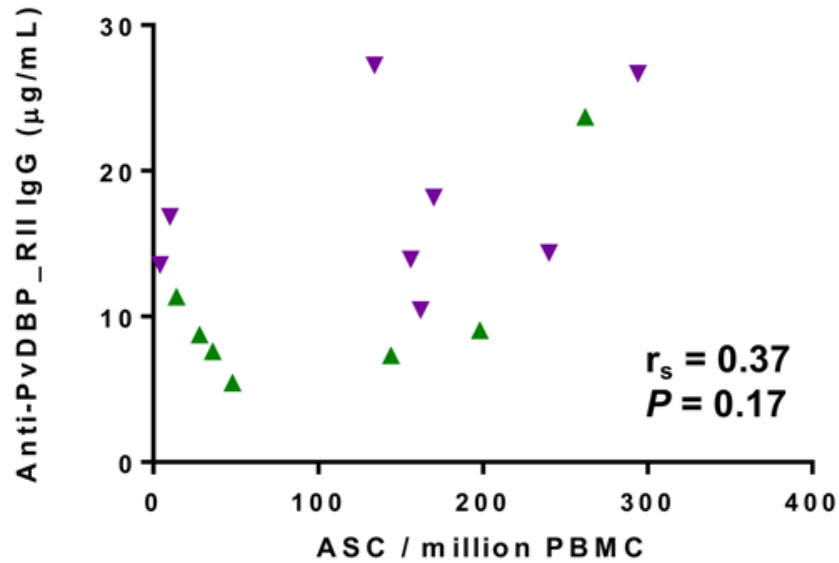

D

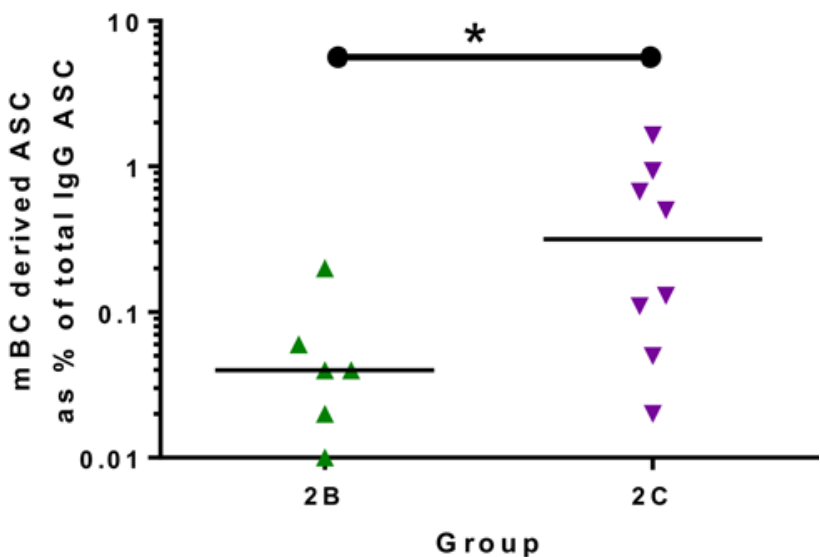

$\mathbf{F}$

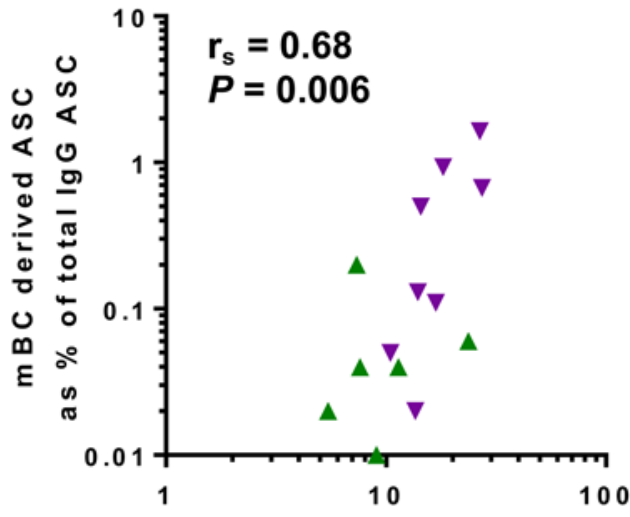

Anti-PvDBP_RII IgG $(\mu \mathrm{g} / \mathrm{mL})$

Figure 5. B cell response to vaccination. (A) PvDBP_RII-specific antibody-secreting cell (ASC) responses were assessed by ex-vivo ELISPOT using PvDBP_RII protein and frozen peripheral blood mononuclear cells (PBMCs) from the day 63 time point. Individual and median responses are shown for each group and reported as PVDBP_RII-specific ASCs per million PBMCs used in the assay. (B) Correlation of the ASC response versus the concentration of serum anti-PvDBP_RII IgG measured at day 84. (C) PvDBP_RII-specific memory B cell (mBC) responses were assessed by ELISPOT assay using PvDBP_RII protein. Frozen PBMCs were thawed and underwent a 6-day polyclonal restimulation during which ASCs were derived from $\mathrm{mBCs}$, before testing in the assay. Individual and median responses are shown from the day 84 time point and are reported as mBCderived PvDBP_RII-specific ASCs per million cultured PBMCs or as (D) percentage of total number of IgG-secreting ASCs. (E and $\mathbf{F}$ ) Correlations of the $\mathrm{mBC}$ response versus the concentration of serum anti-PvDBP_RII IgG at day 84. For all correlations, Spearman's rank correlation coefficient $\left(r_{s}\right)$ and $P$ value are shown. ${ }^{*} P<0.05$. Responses between groups $2 \mathrm{~B}(n=7)$ and $2 \mathrm{C}(n=8)$ were assessed by Mann-Whitney test. PvDBP_RII, region II of the $P$. vivax Duffy-binding protein. 
Table 1. Sequence polymorphisms of PvDBP_RII variants

\begin{tabular}{|c|c|c|c|c|c|c|c|c|c|c|c|c|c|c|c|c|c|c|}
\hline $\begin{array}{l}\text { AA } \\
\text { residue }\end{array}$ & 261 & 263 & 288 & 326 & 330 & 339 & 340 & 341 & 345 & 353 & 359 & 372 & 379 & 392 & 402 & A & 458 & 464 \\
\hline Sall & $\mathbf{F}$ & $\mathbf{R}$ & $\mathbf{L}$ & $\mathbf{K}$ & $\mathbf{N}$ & D & E & $\mathbf{K}$ & $\mathbf{R}$ & $\mathbf{S}$ & $\mathbf{T}$ & $\mathbf{N}$ & $\mathbf{L}$ & W & $\mathbf{S}$ & - & I & E \\
\hline $\mathbf{P}$ & . & $\mathrm{S}$ & $\mathrm{F}$ & . & $D$ & $G$ & $\mathrm{~K}$ & $\mathrm{~N}$ & $\mathrm{H}$ & . & . & $\mathrm{K}$ & 1 & $\mathrm{R}$ & . & . & $\mathrm{K}$ & . \\
\hline 0 & . & $\mathrm{S}$ & . & . & . & G & . & . & $\mathrm{H}$ & . & . & . & 1 & . & K & . & . & . \\
\hline AH & . & $\mathrm{S}$ & . & $E$ & . & $G$ & . & $\mathrm{Q}$ & . & . & . & $\mathrm{K}$ & I & $\mathrm{R}$ & . & . & $\mathrm{K}$ & . \\
\hline HMP013 & $L$ & $\mathrm{~S}$ & . & . & . & $G$ & $\mathrm{~K}$ & $\mathrm{~N}$ & . & $\mathrm{T}$ & $\mathrm{R}$ & . & I & . & . & $\mathrm{L}$ & . & $\mathrm{K}$ \\
\hline
\end{tabular}

The Salvador I (Sall) reference sequence is shown in bold. Amino acid polymorphisms are indicated for the region II of the $P$. vivax Duffy-binding protein (PvDBP_RII) variants (P, O, and AH) described previously (60), plus the $P$. vivax HMP013 strain. Amino acids that are the same as the reference sequence are indicated by a period, and a hyphen indicates an insertion/deletion. ${ }^{A}$ HMP013 has a leucine insertion between V429 and P430 in the Sall reference sequence.

Vaccine-induced antibodies inhibit PvDBP_RII-DARC binding in vitro. We next assessed the ability of vaccineinduced serum IgG to inhibit binding of recombinant vaccine-homologous PvDBP_RII (SalI) to its receptor (in this case the recombinant N-terminal region of DARC), using an in vitro ELISA methodology in Oxford. Day 84 sera were tested using a 2-fold dilution series starting at 1:5 and through to 1:640, with percentage binding inhibition calculated for each volunteer using their matched day 0 serum sample as the baseline control. Example binding-inhibition curves are shown (Supplemental Figure 7A), and 50\% binding-inhibition titers were interpolated from these data (Figure 6A). One sample in group 2A showed a weak $50 \%$ binding-inhibition titer of 1:16. All samples from groups $2 \mathrm{~B}$ and $2 \mathrm{C}$ showed binding inhibition with median $50 \%$ titers of $1: 137$ (range 1:14-1:248) and 1:168 (range 1:52-1:352), respectively. To further assess the quality of the vaccineinduced antibody response, these titers were used to calculate the concentration of anti-PvDBP_RII polyclonal IgG that gives $50 \%$ binding inhibition in each individual (Figure $6 \mathrm{~B}$ ). Across all groups, the median levels were comparable, requiring 128,96 , and $105 \mathrm{ng} / \mathrm{ml}$ in groups $2 \mathrm{~A}, 2 \mathrm{~B}$, and $2 \mathrm{C}$, respectively. However, there was over a 10-fold range across all 16 individuals, with the best responder (in group $2 \mathrm{C}$ ) only requiring $39 \mathrm{ng} / \mathrm{ml}$, versus the worst responder requiring $540 \mathrm{ng} / \mathrm{ml}$ (in group $2 \mathrm{~B}$ ). These data suggest that interindividual qualitative differences exist in terms of the binding-inhibitory capacity of the polyclonal vaccine-induced IgG response.

Given that naturally acquired binding-inhibitory anti-PvDBP_RII antibodies can be strain specific (43, 44), we next proceeded to test the day 0 and 84 sera from group 2 against an established panel of recombinant PvDBP_RII alleles (Table 1) using methodology developed at ICGEB, India (Figure 6, C-F). No binding inhibition was observed for any of the day 0 samples against any PvDBP_RII variant. Data for the Sall variant showed very similar results to those observed with the Oxford assay (Spearman's correlation $r_{\mathrm{s}}=0.67, P=0.002, n=19$ ). Day 84 sera also showed similar 50\% binding-inhibition profiles for the 3 other variants of $\mathrm{PvDBP} \_\mathrm{RII}(\mathrm{PvAH}, \mathrm{PvO}$, and $\mathrm{PvP})$, with the same sample positive in group 2A, and median $50 \%$ binding-inhibition titers greater than $1: 100$ for both groups $2 \mathrm{~B}$ and $2 \mathrm{C}$ for all test variants. At the individual level, all samples showed binding inhibition against each variant of PvDBP_RII, but the $50 \%$ binding-inhibition titers were variable, again consistent with qualitative differences in each polyclonal response (Supplemental Figure 7B). Interestingly, the individual titers were frequently highest against the vaccine-heterologous $\mathrm{PvAH}$ or $\mathrm{PvO}$ alleles.

Finally we tested the day 84 sera against an allele of PvDBP_RII present in the HMP013 Indian strain of $P$. vivax, which has recently been cryobanked for use as an inoculum in blood-stage CHMI clinical trials (16). After generating a draft assembly of HMP013 (see supplementary material), analysis of the PvDBP_ RII sequence from this strain (Table 1) revealed 10 polymorphic positions, of which 5 were not shared with the other variants tested in this study, including some in subdomain 2 (SD2) close to the site shown to bind to aa 19-30 of DARC (Figure 7A). Recombinant PvDBP_RII (HMP013) was subsequently generated and used in the Oxford assay. Binding-inhibition curves were similar to those previously observed with the SalI allele (Figure 7B). Fifty percent binding-inhibition titers were interpolated (Figure 7C), with the data for group 2 again showing a similar profile to those observed with the SalI variant (Figure 7D).

\section{Discussion}

This phase Ia dose-escalation and safety study reports the first data in humans for a vaccine targeting the PvDBP_RII antigen from the blood-stage $P$. vivax malaria parasite. We have shown in healthy malaria-naive 
A

S a II

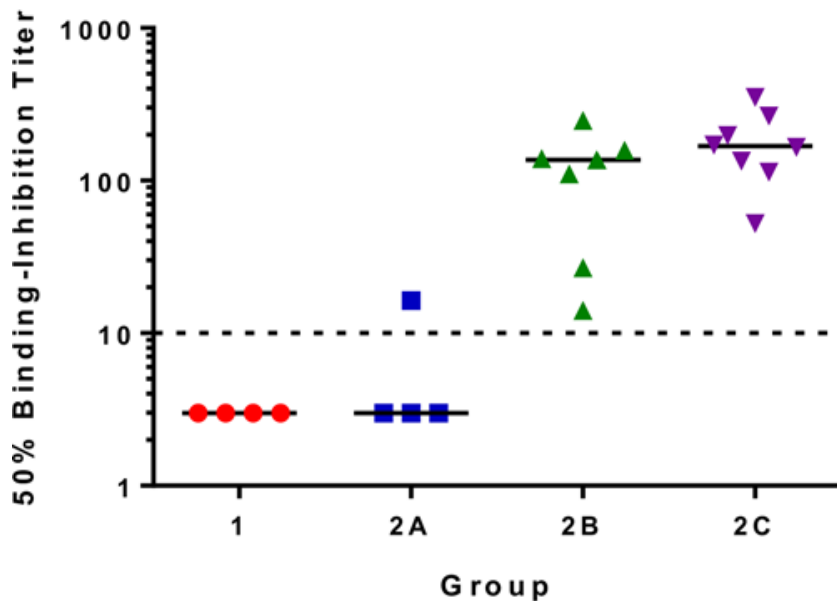

C

S all

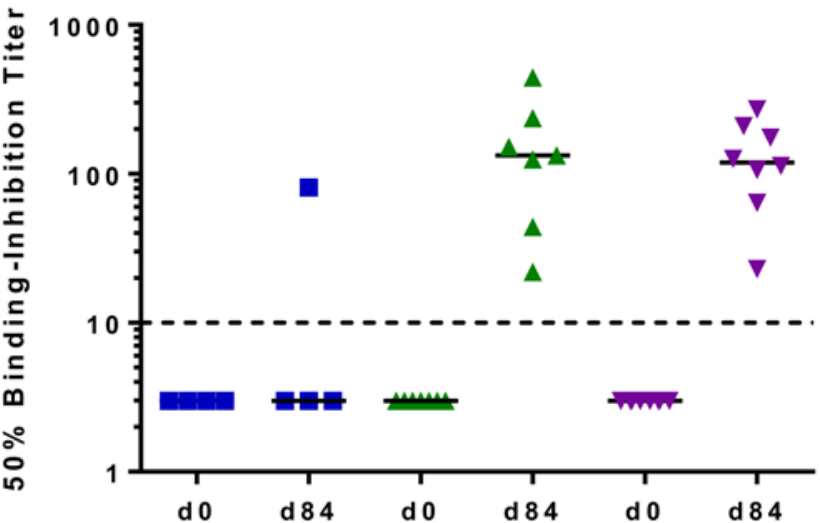

E

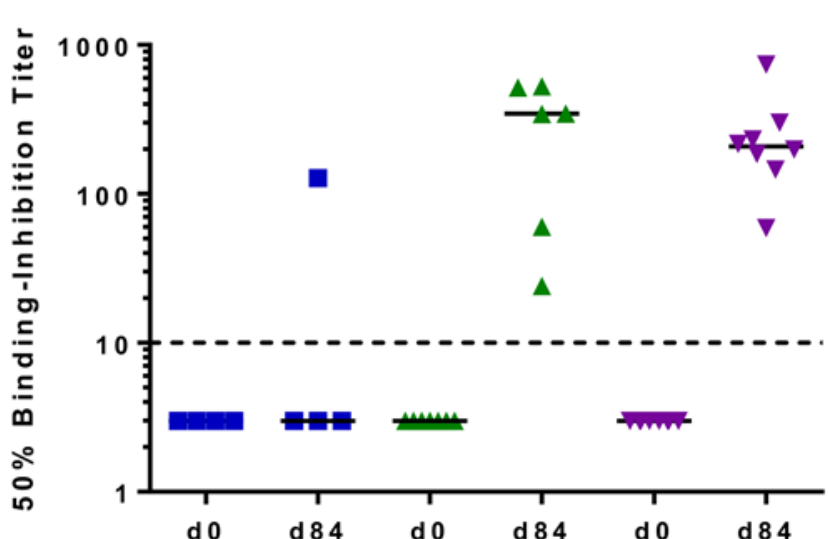

B

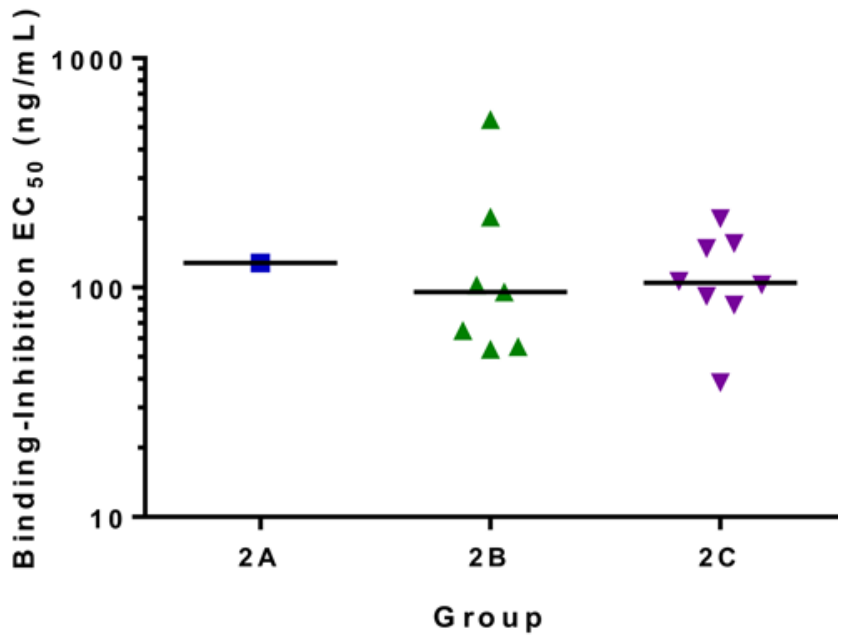

D

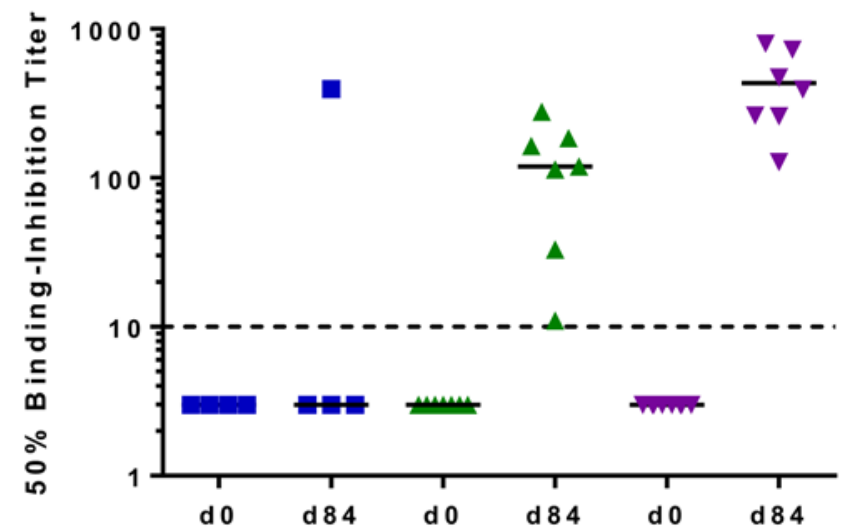

$\mathbf{F}$

Figure 6. PvDBP_RII-DARC in vitro binding inhibition. (A) Day 84 sera from volunteers in groups $1(n=4), 2 \mathrm{~A}(n=4), 2 \mathrm{~B}(n=7)$, and $2 \mathrm{C}(n=8)$ were tested for their ability to inhibit binding of recombinant PvDBP_RII (Sall) to the Duffy antigen receptor for chemokines (DARC) using an ELISA-based assay in Oxford. Samples were titrated starting at 1:5 dilution down to 1:640 (Supplemental Figure 7A). Data show the interpolated dilution for each sample that gave $50 \%$ binding inhibition. (B) For positive samples in $\mathbf{A}(n=16)$, the concentration of anti-PvDBP_RII (Sall) serum IgC that gives $50 \%$ binding inhibition $\left(\mathrm{EC}_{50}\right.$ ) was calculated by dividing the serum ELISA $\mu \mathrm{g} / \mathrm{ml}$ by the $50 \%$ binding-inhibition serum titer. The result is reported in $\mathrm{ng} / \mathrm{ml}$. (C-F) Day 0 and day 84 sera were assessed as in $\mathbf{A}$ using the assay established at ICGEB, India, using 4 recombinant alleles of PvDBP_RII: Sall, PvAH, PvO, and PvP. In all panels, the individual and median results are shown for each group. The dashed line shows an arbitrary cutoff below which negative samples are plotted. PvDBP_RII, region II of the P. vivax Duffy-binding protein; Sall, Salvador I reference strain. 


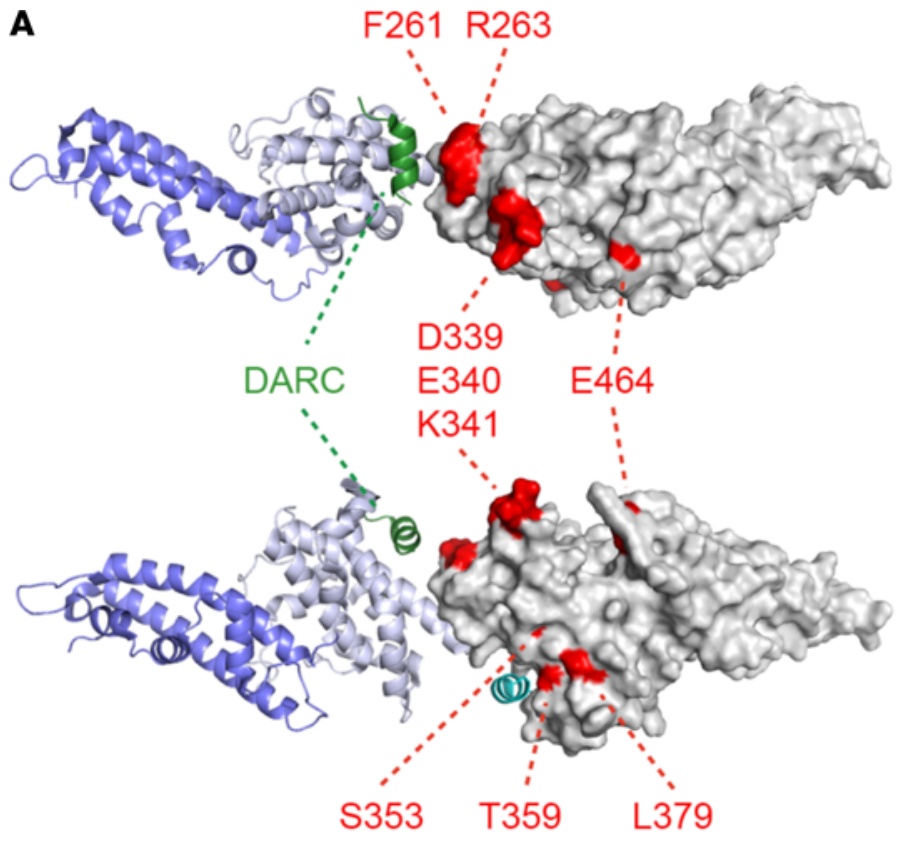

C

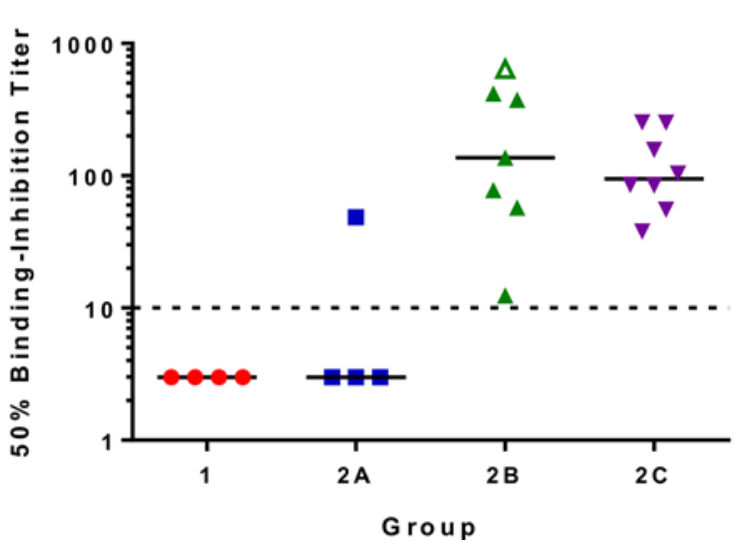

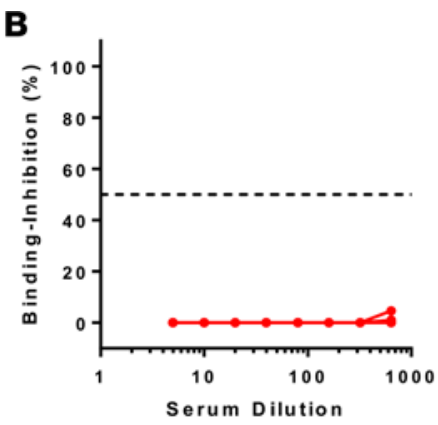
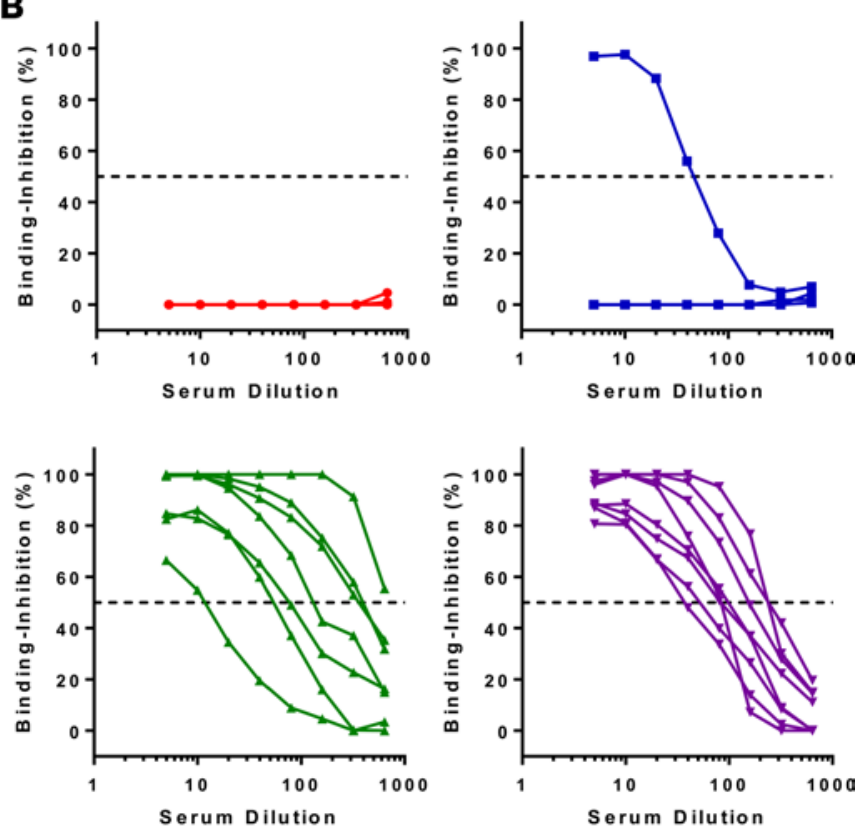

D

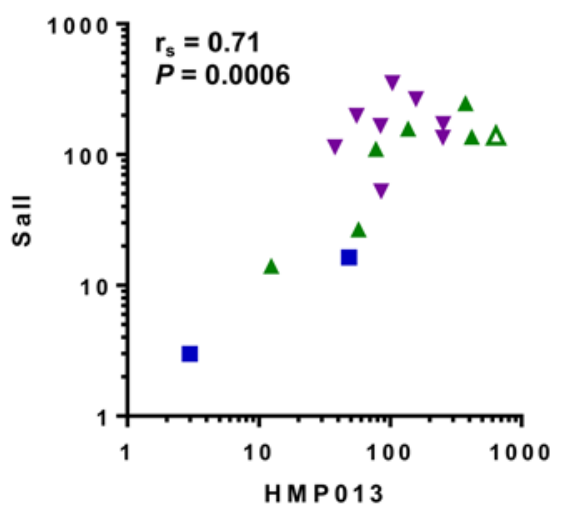

Figure 7. Binding inhibition of the P. vivax HMP013 strain DBP_RII. (A) The location of polymorphic residues in PvDBP_RII (HMP013 strain) have been marked on a structure of the PVDBP_RII (Sall strain) dimer bound to the Duffy antigen receptor for chemokines (DARC) aa 19-30 (PDB code 4NVU) (24). Two views of the dimer are shown, rotated by 90 degrees around the horizontal axis. One molecule of PvDBP_RII is shown in gray surface representation with polymorphic residues colored in red. The second molecule of PVDBP_RII is in blue cartoon representation with SD3 in a darker blue. The 2 helices from DARC are shown in green and cyan, respectively. (B) Day 84 sera from volunteers in groups $1(n=4), 2 \mathrm{~A}(n=4), 2 \mathrm{~B}(n=7)$, and 2C ( $n=8)$ were tested for their ability to inhibit binding of recombinant PVDBP_RII (HMP013) to DARC using the ELISA-based assay in Oxford. Samples were titrated starting at 1:5 dilution down to 1:640. Dashed line indicates 50\% binding inhibition. Groups coded by color and symbol. (C) Data show the interpolated dilution for each sample that gave $50 \%$ binding inhibition. One sample in group 2B did not reach 50\% binding inhibition by 1:640 dilution and is plotted at this final titer with open triangle symbol. (D) Correlation of 50\% binding-inhibition titers for the Sall and HMP013 alleles of PvDBP_RII measured using the assay in Oxford. Spearman's rank correlation coefficient $\left(r_{s}\right)$ and $P$ value are shown $(n=19)$. PvDBP_RII, region II of the $P$. vivax Duffy-binding protein.

adult volunteers that a recombinant ChAd63-MVA heterologous prime-boost immunization regimen can induce binding-inhibitory antigen-specific serum antibody responses in addition to $\mathrm{B}$ and $\mathrm{T}$ cell responses. ChAd63 and MVA recombinant for PvDBP_RII also demonstrated a favorable safety profile. Reactogenicity of the ChAd63 PvDBP_RII vector was similar to that seen consistently with the same doses of ChAd63 vectored vaccines encoding the $P$. falciparum pre-erythrocytic malaria antigens ME-TRAP or PfCSP (35, 36) and the blood-stage antigens PfMSP1 or PfAMA1 (37, 38, 42, 45). In more recent years, safety and immunogenicity data for the ME-TRAP vaccines have been reported in adults, children, and infants residing in malaria-endemic areas (46). Our data with ChAd63 PvDBP_RII add to the growing body of evidence that this simian adenovirus vector is safe for clinical use. Reactogenicity of the MVA PvDBP_RII vector 
appeared to be more pronounced than the ChAd63 vector and increased with dose, again consistent with previous experience of using this orthopoxvirus vector for $P$. falciparum vaccines $(37,38)$ as well as other disease targets such as respiratory syncytial virus (47), hepatitis C virus (48), Ebola virus (40), HIV, and Mycobacterium tuberculosis (49). Indeed, the clinical safety of MVA as a recombinant vaccine vector for many infectious diseases and cancer is now well documented.

The ChAd63-MVA delivery platform was originally developed to induce $\mathrm{T}$ cell responses against a target blood-stage malaria antigen in humans $(32,33)$. Similar to our data in mice using this vaccine (27), the results presented here show that PvDBP_RII-specific IFN- $\gamma$ T cell responses were induced and peaked at median levels of greater than 2,000 SFU/million PBMCs following the MVA boost. The kinetics and magnitude of this response are extremely similar to those previously seen with the same vectors encoding $P$. falciparum antigens (35-38). These previous studies using the ChAd63-MVA regimen, as well as other studies using alternative ChAd serotypes followed by MVA boost $(40,47,48)$, have routinely shown that a mixed antigen-specific $\mathrm{CD} 4^{+} / \mathrm{CD}^{+} \mathrm{T}$ cell response is induced in humans.

The ELISPOT data showed that the IFN- $\gamma$ T cell responses were spread across all 6 peptide pools spanning the PvDBP_RII antigen. A previous study assessed IFN- $\gamma$ and IL-10 T cell responses to aa 291-460 of PvDBP_RII in naturally exposed children and adults from Papua New Guinea. These data showed that age-dependent low-level responses are detectable in a subset of individuals following natural $P$. vivax infection (<150 SFU/million PBMCs using a 3-day cultured ELISPOT protocol, as opposed to the overnight stimulation used in the ex vivo assay reported here) (50). Five PvDBP_RII T cell epitopes were identified by peptide mapping, with 3 of these containing polymorphic residues leading to variant-specific cellular responses (50). Nevertheless, the contribution of $\mathrm{T}$ cell responses to blood-stage immunity against $P$. vivax remains unclear. In the case of $P$. falciparum, clinical trials using whole-parasite immunization (51) or ChAd63-MVA vectors encoding PfMSP1 or PfAMA1 (45) failed to show an impact on blood-stage parasite growth following CHMI despite strong $\mathrm{T}$ cell induction by vaccination. However, recent data from other CHMI studies show that, unlike $P$. falciparum, blood-stage $P$. vivax activates cytotoxic $\mathrm{CD} 38^{+} \mathrm{CD} 8^{+} \mathrm{T}$ cells that could target parasites residing within MHC class I-expressing reticulocytes (52), suggesting that it may be possible for effector $\mathrm{T}$ cells to play a more direct role against this species of human malaria parasite.

In agreement with preclinical data in mice and rabbits (27), the ChAd63-MVA prime-boost regimen also induced PvDBP_RII-specific serum IgG antibody responses, peaking at a median of $0.3 \mu \mathrm{g} / \mathrm{ml} \mathrm{after}$ ChAd63 prime and $15.6 \mu \mathrm{g} / \mathrm{ml}$ after MVA boost in the full-dose vaccination groups. The kinetics and magnitude of the antigen-specific IgG, ASC, and $\mathrm{mBC}$ responses induced here in malaria-naive humans are consistent with those reported for the same vectors encoding the $P$. falciparum blood-stage antigens PfMSP1 and PfAMA1 $(37,38,41,42)$. With regard to the PvDBP_RII-specific antibody concentrations, these were lower than those seen following ChAd63-MVA immunization with PfAMA1 (37, 42) and PfMSP1 (37), but 8 -fold higher than with PfCSP (36). Similar to these $P$. falciparum vaccines $(42,53)$, the anti-PvDBP RII serum IgG response was largely composed of IgG1 and some IgG3, with moderate avidity as measured by NaSCN-displacement ELISA. These qualitative aspects of the vaccine-induced antibody responses are consistent with those observed to the same antigen following natural infection in endemic populations (54, 55); however, the contributions of antibody isotype, affinity, and avidity to protection against the $P$. vivax merozoite remain poorly understood.

Studies of naturally acquired immunity following $P$. vivax exposure have reported the induction of strain-specific immunity $(43,44)$ and numerous sequence polymorphisms, consistent with immune evasion, have been found within the PvDBP_RII antigen, with the majority localized to SD2 $(24,56)$. Nevertheless, high-titer naturally acquired BIAbs that block binding of diverse PvDBP_RII alleles from $P$. vivax field isolates have also been reported, albeit at low frequency $(30,31)$. Once acquired, these antibodies are maintained and associate with clinical immunity to $P$. vivax. In contrast to these epidemiological data, preclinical immunogenicity studies with the SalI allele of PvDBP_RII have shown that this immunogen is capable of eliciting high-titer, cross-reactive BIAbs, as assessed using the ELISA-based binding-inhibition assay (28). Consistent with these data, our studies here suggest that PvDBP_RII vaccination of humans can elicit antibodies that qualitatively differ from those induced by natural exposure. Across all vaccinees who received the ChAd63-MVA regimen, anti-PvDBP_RII responses were induced that blocked binding of variant PvDBP_RII alleles to DARC in vitro including one from the HMP013 strain, suggesting this strain would be suitable to test vaccine efficacy in a future phase IIa CHMI study (16). Encouragingly, median $50 \%$ binding-inhibition titers greater than 1:100 were consistently observed for all test variants; these are 
higher than the $\sim 1: 20$ titers reported by others using a similar assay for naturally acquired, strain-transcending BIAbs in a limited number of children in Papua New Guinea and associated with clinical immunity (30). However, this association with clinical immunity has never been formally demonstrated in the context of a vaccine clinical trial. ChAd63-MVA PvDBP_RII is the first candidate vaccine against blood-stage $P$. vivax to reach clinical testing. It is therefore vital in a future CHMI efficacy study to assess whether vaccineinduced BIAbs associate with control of blood-stage parasite growth.

The data obtained from this study also suggested interindividual qualitative differences in terms of the polyclonal anti-PvDBP_RII IgG response, as would be anticipated following human vaccination. A recent cohort study in the Brazilian Amazon has suggested that genetic variation in HLA class II genes can influence antibody responses against PvDBP_RII following natural $P$. vivax infection (57). Similarly, studies of naturally acquired anti-PvDBP_RII IgG responses (58) as well as mouse monoclonal antibodies $(59,60)$ have reported linear and conformational epitopes. Here we failed to detect linear responses by ELISA using a peptide array, and our ongoing work will focus on elucidating epitopes recognized by vaccine-induced human B cells in order to guide future immuno-monitoring. Further ongoing work is seeking to assess antibody function against $P$. vivax parasites. Importantly, we have previously reported that adenovirus-MVA immunization of mice and rabbits elicits antibodies that recognize native parasite antigen by immunofluorescence assay (IFA) (27). Future studies will focus on optimizing short-term invasion-inhibition assay methodology (61) to allow for functional testing of vaccine-induced antibodies from human volunteers in clinical trials.

Overall, the association between Duffy negativity and protection against blood-stage $P$. vivax infection was first reported in 1976 (15), but until now this observation has not been translated into a clinical vaccine candidate. The intervening years have seen the PvDBP_RII-DARC interaction described in molecular detail and related immuno-epidemiology extensively studied in the field. Here we extend this work and demonstrate, possibly for the first time, that substantial PvDBP_RII-specific antibodies as well as B cell and $\mathrm{T}$ cell responses can be induced safely by immunization in humans, using a leading viral vectored delivery strategy that is in clinical development for numerous difficult and emerging diseases and cancer. Encouragingly for the $P$. vivax vaccine field, a second PvDBP_RII protein-based vaccine formulated in the emulsified version of glucopyranosyl lipid adjuvant (GLA-SE) has also recently entered a phase I clinical trial in India (CTRI/2016/09/007289). The demonstration in parallel of a blood-stage CHMI model for vaccine testing using $P$. falciparum (62), and the banking of similar blood-stage inocula for $P$. vivax (16), should allow for this ChAd63-MVA vaccine and others to progress to rapid phase IIa proof-of-concept efficacy testing in the near future.

\section{Methods}

Detailed methods are provided in supplemental methods.

ChAd63 and MVA PvDBP_RII vaccines. The design, production, and preclinical testing of the viral vector vaccines have been reported previously (27). Briefly, both recombinant viruses express the same 984-bp coding sequence of PvDBP_RII from the SalI strain of P. vivax, aa D194-T521 (GenBank Accession DQ156512). ChAd63 PvDBP_RII was manufactured under current Good Manufacturing Practice (cGMP) conditions by the Clinical Biomanufacturing Facility (CBF), University of Oxford, UK, and MVA PvDBP_RII was manufactured under cGMP conditions by IDT Biologika GmbH, Germany, both as previously described (37).

Study design and approvals. The VAC051 study was a phase Ia open-label, dose-escalation, first-inhuman, nonrandomized trial of the viral vectored vaccines ChAd63 PvDBP_RII and MVA PvDBP_RII given in a prime-boost regimen with an 8-week interval. The study was conducted at the Centre for Clinical Vaccinology and Tropical Medicine (CCVTM), University of Oxford, Oxford, UK. The study received ethical approval from the Oxfordshire Research Ethics Committee A in Oxford, UK (REC reference 13/ SC/0001). The study was also reviewed and approved by the UK Medicines and Healthcare products Regulatory Agency (MHRA, reference 21584/0312/001-0001). Volunteers signed written consent forms and consent was verified before each vaccination. The trial was registered on Clinicaltrials.gov (NCT01816113) and was conducted according to the principles of the current revision of the Declaration of Helsinki 2008 and in full conformity with the ICH guidelines for Good Clinical Practice (GCP). The primary endpoint of the study was to assess the safety of ChAd63 PvDBP_RII and MVA PvDBP_RII, with a secondary endpoint to assess immunogenicity. 
Participants. Healthy, malaria-naive males and nonpregnant females aged 18-50 were invited to participate in the study. All volunteers were recruited and vaccinated at the CCVTM, part of the Oxford Vaccine Centre (OVC), at the University of Oxford. Twenty-four volunteers were enrolled in total. A full list of inclusion and exclusion criteria is reported in the supplemental methods.

Safety analysis. Data on AEs were collected throughout a volunteer's participation in the trial, either on the diary cards they were issued with following vaccination or at follow-up visits. Any solicited AEs occurring during the diary card period were defined as being at least possibly related to vaccination. The likely causality of all other AEs was assessed as described in the protocol and all AEs considered possibly, probably, or definitely related to vaccination are reported (Supplemental Table 1). Further details on grading are provided in the supplemental material.

Peptides. Peptides for ex-vivo IFN- $\gamma$ ELISPOT were purchased from NEO Peptide (Supplemental Table 2), and for peptide ELISAs, biotinylated 20-mer peptides were synthesized by Mimotopes (Supplemental Table 3).

Recombinant PvDBP_RII and DARC proteins. Recombinant PvDBP_RII (SalI) protein for ELISA-based assays was generated using a Drosophila melanogaster Schneider 2 (S2) polyclonal stable cell line (ExpreS ${ }^{2}$ platform, ExpreS²ion Biotechnologies) (63). Recombinant N-terminal DARC was produced for use in the PvDBP_RII-DARC binding-inhibition assay in Oxford, UK, by transient transfection of suspension HEK293E cells grown in EXPI293 expression medium (Thermo Fisher Scientific) (34). For the HMP013 strain of $P$. vivax, the sequence of the PvDBP_RII gene was verified by Sanger sequencing using primers listed in Supplemental Table 4, and recombinant PvDBP_RII (HMP013) produced in suspension EXPI293F cells (Thermo Fisher Scientific) by transient transfection.

Ex vivo IFN- $\gamma$ ELISPOT. Ex vivo IFN- $\gamma$ ELISPOT was used to assess the kinetics and magnitude of the vaccine-induced $\mathrm{T}$ cell responses over time. Fresh PBMCs were used in all assays using a previously described protocol (38). Results are expressed as IFN- $\gamma$ SFU per million PBMCs.

Total IgG ELISAs. ELISAs were performed using standardized methodology as previously described (37, 38), except that plates were coated with recombinant PvDBP_RII protein produced from the Drosophila S2 cells and blocked with StartingBlock T20 solution (Thermo Fisher Scientific). Responses measured in AU are reported in $\mu \mathrm{g} / \mathrm{ml}$ following generation of a conversion factor by CFCA.

Avidity and isotype ELISAs. IgG antibody avidity was assessed by NaSCN-displacement ELISA using previously described methodology (53), except that plates were coated with recombinant PvDBP_RII produced from the Drosophila S2 cells at $2 \mu \mathrm{g} / \mathrm{ml}$ and blocked with StartingBlock T20 solution. The concentration of $\mathrm{NaSCN}$ required to reduce the $\mathrm{OD}_{405}$ to $50 \%$ of that without $\mathrm{NaSCN}$ was used as a measure of avidity $\left(\mathrm{IC}_{50}\right)$. Antibody isotype ELISAs were also performed using methodology described in detail elsewhere (53) with the same exceptions as for the avidity ELISA.

$m B C$ and ASC ELISPOT. mBC ELISPOT assays were performed as described in detail elsewhere (41). Ex vivo ASC ELISPOT assays were performed using frozen PBMCs directly prepared and added to the ELISPOT plate with no preceding 6-day culture.

PvDBP_RII-DARC binding-inhibition assay. Sera were tested for their ability to inhibit binding of recombinant PvDBP_RII to DARC using an assay developed at Oxford, UK (Figure 6, A and B; Figure 7, B-D; and Supplemental Figure 7A). Sera were also tested using a similar assay previously established at ICGEB, India (64) (Figures 6, C-F and Supplemental Figure 7B). Four variants of recombinant PvDBP_RII (SalI, PvAH, PvO, and PvP) were used.

Statistics. Data were analyzed using GraphPad Prism version 6.07 for Windows. All tests were 2-tailed and are described in the text. A value of $P$ less than 0.05 was considered significant.

\section{Author contributions}

ROP, SES, SCE, KHM, TAR, DL, ARS, GML, NJE, IDP, RF, DGWA, SCdC, AVSH, CEC, and SJD conceived and performed the experiments. ROP, SES, SCE, KHM, TAR, DL, MKH, CEC, and SJD analyzed the data. JJ, TJ, JSM, WAdJ, AN, SM, EB, and CEC contributed reagents, materials, and analysis tools. TDO assembled and annotated the genome sequence. RR and AML managed the project. ROP and SJD wrote the manuscript.

\section{Acknowledgments}

The authors are grateful for the assistance of Julie Furze, Fay Nugent, Susanne Hodgson, Sharon Sheehan, Alexandra Spencer, Sumi Biswas, Alexander Douglas, Mary Smith, Adrienne Cook, Raquel Lopez-Ramon, 
Sam French, Natalie Lella, and Sylwester Pawluk (Jenner Institute, University of Oxford); Carly Banner for arranging contracts (University of Oxford); Matt Berriman and Mandy Sanger for the sequencing of the HMP013 isolate (Wellcome Trust Sanger Institute); Yves Durocher for provision of HEK293E cells (CNRCNRC, Canada); David Staunton (Biophysical Instrument Facility, Department of Biochemistry, University of Oxford); Gemma Harris (Rutherford Appleton Laboratory, Harwell Campus, Didcot, UK); Okairòs and GSK for provision of the ChAd63 vector; and all the study volunteers.

This work was supported by a UK Medical Research Council (MRC) grant (number G1100086). The study was also supported in part by UK National Institute of Health Research (NIHR) infrastructure through the NIHR Oxford Biomedical Research Centre and the Wellcome Trust (084113/Z/07/Z). DL was supported by the Rhodes Trust. TAR holds a Wellcome Trust Research Training Fellowship (108734/Z/15/Z). SCdC was a $\mathrm{PhD}$ student supported by the European Malaria Vaccine Development Association, a European Commission Framework Programme 6-funded consortium (grant LSHP-CT-2007-037506). TDO is supported by the Wellcome Trust (WT 098051). JSM is supported by an NHMRC Practitioner Fellowship (number 1041802). AVSH and SJD are Jenner Investigators. SJD is a Lister Institute Research Prize Fellow and a Wellcome Trust Senior Fellow (grant number 106917/Z/15/Z).

Address correspondence to: Ruth O. Payne or Simon J. Draper, Jenner Institute, Old Road Campus Research Building, Roosevelt Drive, Oxford, OX3 7DQ, United Kingdom. Phone: 44.1865.617624; Email: ruth.payne@ndm.ox.ac.uk (R.O. Payne); simon.draper@ndm.ox.ac.uk (S.J. Draper).

1. Halbroth BR, Draper SJ. Recent developments in malaria vaccinology. Adv Parasitol. 2015;88:1-49.

2. Gething PW, et al. A long neglected world malaria map: Plasmodium vivax endemicity in 2010. PLoS Negl Trop Dis. 2012;6(9):e1814.

3. Howes RE, et al. Plasmodium vivax transmission in Africa. PLoS Negl Trop Dis. 2015;9(11):e0004222.

4. Baird JK. Evidence and implications of mortality associated with acute Plasmodium vivax malaria. Clin Microbiol Rev. 2013;26(1):36-57.

5. Moorthy VS, Newman RD, Okwo-Bele JM. Malaria vaccine technology roadmap. Lancet. 2013;382(9906):1700-1701.

6. Herrera S, et al. Phase I safety and immunogenicity trial of Plasmodium vivax CS derived long synthetic peptides adjuvanted with montanide ISA 720 or montanide ISA 51. Am J Trop Med Hyg. 2011;84(2 Suppl):12-20.

7. Bennett JW, et al. Phase 1/2a trial of Plasmodium vivax malaria vaccine candidate VMP001/AS01B in malaria-naive adults: safety, immunogenicity, and efficacy. PLoS Negl Trop Dis. 2016;10(2):e0004423.

8. Vanloubbeeck Y, et al. Comparison of the immune responses induced by soluble and particulate Plasmodium vivax circumsporozoite vaccine candidates formulated in AS01 in rhesus macaques. Vaccine. 2013;31(52):6216-6224.

9. Malkin EM, et al. Phase 1 vaccine trial of Pvs25H: a transmission blocking vaccine for Plasmodium vivax malaria. Vaccine. 2005;23(24):3131-3138.

10. Wu Y, et al. Phase 1 trial of malaria transmission blocking vaccine candidates Pfs 25 and Pvs 25 formulated with montanide ISA 51. PLoS One. 2008;3(7):e2636.

11. Mueller I, Shakri AR, Chitnis CE. Development of vaccines for Plasmodium vivax malaria. Vaccine. 2015;33(52):7489-7495.

12. Tham WH, Healer J, Cowman AF. Erythrocyte and reticulocyte binding-like proteins of Plasmodium falciparum. Trends Parasitol. 2012;28(1):23-30.

13. Malleret B, et al. Plasmodium vivax: restricted tropism and rapid remodeling of CD71-positive reticulocytes. Blood. 2015;125(8):1314-1324.

14. Chitnis CE, Sharma A. Targeting the Plasmodium vivax Duffy-binding protein. Trends Parasitol. 2008;24(1):29-34

15. Miller LH, Mason SJ, Clyde DF, McGinniss MH. The resistance factor to Plasmodium vivax in blacks. The Duffy-blood-group genotype, FyFy. N Engl J Med. 1976;295(6):302-304.

16. Payne RO, Griffin PM, McCarthy JS, Draper SJ. Plasmodium vivax controlled human malaria infection - progress and prospects. Trends Parasitol. 2017;33(2):141-150.

17. Singh AP, Ozwara H, Kocken CH, Puri SK, Thomas AW, Chitnis CE. Targeted deletion of Plasmodium knowlesi Duffy binding protein confirms its role in junction formation during invasion. Mol Microbiol. 2005;55(6):1925-1934.

18. Zimmerman PA, Ferreira MU, Howes RE, Mercereau-Puijalon O. Red blood cell polymorphism and susceptibility to Plasmodium vivax. Adv Parasitol. 2013;81:27-76.

19. Tham WH, Beeson JG, Rayner JC. Plasmodium vivax vaccine research - we've only just begun. Int J Parasitol. 2017;47(2-3):111-118.

20. Menard D, et al. Whole genome sequencing of field isolates reveals a common duplication of the Duffy binding protein gene in Malagasy Plasmodium vivax strains. PLoS Negl Trop Dis. 2013;7(11):e2489.

21. Hester J, et al. De novo assembly of a field isolate genome reveals novel Plasmodium vivax erythrocyte invasion genes. $P L o S$ Negl Trop Dis. 2013;7(12):e2569.

22. Hostetler JB, et al. Independent origin and global distribution of distinct Plasmodium vivax Duffy binding protein gene duplications. PLoS Negl Trop Dis. 2016;10(10):e0005091.

23. Ntumngia FB, Thomson-Luque R, Torres Lde M, Gunalan K, Carvalho LH, Adams JH. A Novel erythrocyte binding protein of Plasmodium vivax suggests an alternate invasion pathway into Duffy-positive reticulocytes. MBio. 2016;7(4):e01261. 
24. Batchelor JD, Malpede BM, Omattage NS, DeKoster GT, Henzler-Wildman KA, Tolia NH. Red blood cell invasion by Plasmodium vivax: structural basis for DBP engagement of DARC. PLoS Pathog. 2014;10(1):e1003869.

25. Batchelor JD, Zahm JA, Tolia NH. Dimerization of Plasmodium vivax DBP is induced upon receptor binding and drives recognition of DARC. Nat Struct Mol Biol. 2011;18(8):908-914.

26. Moreno A, et al. Preclinical assessment of the receptor-binding domain of Plasmodium vivax Duffy-binding protein as a vaccine candidate in rhesus macaques. Vaccine. 2008;26(34):4338-4344.

27. de Cassan SC, et al. Preclinical assessment of viral vectored and protein vaccines targeting the Duffy-binding protein region II of Plasmodium vivax. Front Immunol. 2015;6:348.

28. Wiley SR, et al. Targeting TLRs expands the antibody repertoire in response to a malaria vaccine. Sci Transl Med. 2011;3(93):93ra69.

29. Singh AP, Puri SK, Chitnis CE. Antibodies raised against receptor-binding domain of Plasmodium knowlesi Duffy binding protein inhibit erythrocyte invasion. Mol Biochem Parasitol. 2002;121(1):21-31.

30. King CL, et al. Naturally acquired Duffy-binding protein-specific binding inhibitory antibodies confer protection from bloodstage Plasmodium vivax infection. Proc Natl Acad Sci USA. 2008;105(24):8363-8368.

31. Nicolete VC, Frischmann S, Barbosa S, King CL, Ferreira MU. Naturally acquired binding-inhibitory antibodies to Plasmodium vivax Duffy binding protein and clinical immunity to malaria in rural Amazonians. J Infect Dis. 2016;214(10):1539-1546.

32. de Cassan SC, Draper SJ. Recent advances in antibody-inducing poxviral and adenoviral vectored vaccine delivery platforms for difficult disease targets. Expert Rev Vaccines. 2013;12(4):365-378.

33. Draper SJ, et al. Effective induction of high-titer antibodies by viral vector vaccines. Nat Med. 2008;14(8):819-821.

34. Douglas AD, et al. A PfRH5-based vaccine is efficacious against heterologous strain blood-stage Plasmodium falciparum infection in aotus monkeys. Cell Host Microbe. 2015;17(1):130-139.

35. O'Hara GA, et al. Clinical assessment of a recombinant simian adenovirus ChAd63: a potent new vaccine vector. J Infect Dis. 2012;205(5):772-781

36. de Barra E, et al. A phase Ia study to assess the safety and immunogenicity of new malaria vaccine candidates ChAd63 CS administered alone and with MVA CS. PLoS One. 2014;9(12):e115161.

37. Sheehy SH, et al. Phase Ia clinical evaluation of the Plasmodium falciparum blood-stage antigen MSP1 in ChAd63 and MVA vaccine vectors. Mol Ther. 2011;19(12):2269-2276.

38. Sheehy SH, et al. Phase Ia clinical evaluation of the safety and immunogenicity of the Plasmodium falciparum blood-stage antigen AMA1 in ChAd63 and MVA vaccine vectors. PLoS ONE. 2012;7(2):e31208.

39. Sedegah M, et al. Adenovirus 5-vectored P. falciparum vaccine expressing CSP and AMA1. Part A: safety and immunogenicity in seronegative adults. PLoS One. 2011;6(10):e24586.

40. Ewer K, et al. A monovalent chimpanzee adenovirus ebola vaccine boosted with MVA. N Engl J Med. 2016;374(17):1635-1646.

41. Elias SC, et al. Analysis of human B-cell responses following ChAd63-MVA MSP1 and AMA1 immunization and controlled malaria infection. Immunology. 2014;141(4):628-644.

42. Hodgson $\mathrm{SH}$, et al. Combining viral vectored and protein-in-adjuvant vaccines against the blood-stage malaria antigen AMA1: report on a phase 1a clinical trial. Mol Ther. 2014;22(12):2142-2154.

43. Cole-Tobian JL, et al. Strain-specific duffy binding protein antibodies correlate with protection against infection with homologous compared to heterologous plasmodium vivax strains in Papua New Guinean children. Infect Immun. 2009;77(9):4009-4017.

44. Ceravolo IP, et al. Naturally acquired inhibitory antibodies to Plasmodium vivax Duffy binding protein are short-lived and allele-specific following a single malaria infection. Clin Exp Immunol. 2009;156(3):502-510.

45. Sheehy SH, et al. ChAd63-MVA-vectored blood-stage malaria vaccines targeting MSP1 and AMA1: assessment of efficacy against mosquito bite challenge in humans. Mol Ther. 2012;20(12):2355-2368.

46. Afolabi MO, et al. Safety and immunogenicity of ChAd63 and MVA ME-TRAP in West African children and infants. Mol Ther. 2016;24(8):1470-1477

47. Green CA, et al. Chimpanzee adenovirus- and MVA-vectored respiratory syncytial virus vaccine is safe and immunogenic in adults. Sci Transl Med. 2015;7(300):300ra126.

48. Swadling L, et al. A human vaccine strategy based on chimpanzee adenoviral and MVA vectors that primes, boosts, and sustains functional HCV-specific T cell memory. Sci Transl Med. 2014;6(261):261ra153.

49. Gilbert SC. Clinical development of modified vaccinia virus Ankara vaccines. Vaccine. 2013;31(39):4241-4246.

50. Xainli J, Baisor M, Kastens W, Bockarie M, Adams JH, King CL. Age-dependent cellular immune responses to Plasmodium vivax Duffy binding protein in humans. J Immunol. 2002;169(6):3200-3207.

51. Bijker EM, et al. Protection against malaria after immunization by chloroquine prophylaxis and sporozoites is mediated by preerythrocytic immunity. Proc Natl Acad Sci U S A. 2013;110(19):7862-7867.

52. Burel JG, Apte SH, McCarthy JS, Doolan DL. Plasmodium vivax but not Plasmodium falciparum blood-stage infection in humans is associated with the expansion of a $\mathrm{CD}^{+} \mathrm{T}$ cell population with cytotoxic potential. PLoS Negl Trop Dis. 2016;10(12):e0005031.

53. Biswas S, et al. Assessment of humoral immune responses to blood-stage malaria antigens following ChAd63-MVA immunization, controlled human malaria infection and natural exposure. PLoS One. 2014;9(9):e107903.

54. Tran TM, et al. Comparison of IgG reactivities to Plasmodium vivax merozoite invasion antigens in a Brazilian Amazon population. Am J Trop Med Hyg. 2005;73(2):244-255.

55. Zakeri S, Babaeekhou L, Mehrizi AA, Abbasi M, Djadid ND. Antibody responses and avidity of naturally acquired anti-Plasmodium vivax Duffy binding protein (PvDBP) antibodies in individuals from an area with unstable malaria transmission. Am $J$ Trop Med Hyg. 2011;84(6):944-950.

56. Sampath S, et al. Glycan masking of Plasmodium vivax Duffy Binding Protein for probing protein binding function and vaccine development. PLoS Pathog. 2013;9(6):e1003420

57. Kano FS, et al. The presence, persistence and functional properties of Plasmodium vivax Duffy binding protein ii antibodies are influenced by HLA class II allelic variants. PLoS Negl Trop Dis. 2016;10(12):e0005177.

58. Chootong $\mathrm{P}$, et al. Mapping epitopes of the Plasmodium vivax Duffy binding protein with naturally acquired inhibitory antibodies. 
Infect Immun. 2010;78(3):1089-1095.

59. Chen E, et al. Broadly neutralizing epitopes in the Plasmodium vivax vaccine candidate Duffy binding protein. Proc Natl Acad Sci U S A. 2016;113(22):6277-6282.

60. Ntumngia FB, et al. Conserved and variant epitopes of Plasmodium vivax Duffy binding protein as targets of inhibitory monoclonal antibodies. Infect Immun. 2012;80(3):1203-1208.

61. Russell B, et al. A reliable ex vivo invasion assay of human reticulocytes by Plasmodium vivax. Blood. 2011;118(13):e74-e81.

62. Payne RO, et al. Demonstration of the blood-stage Plasmodium falciparum controlled human malaria infection model to assess efficacy of the P. falciparum apical membrane antigen 1 vaccine, FMP2.1/AS01. J Infect Dis. 2016;213(11):1743-1751.

63. Hjerrild KA, et al. Production of full-length soluble Plasmodium falciparum RH5 protein vaccine using a Drosophila melanogaster Schneider 2 stable cell line system. Sci Rep. 2016;6:30357.

64. Shakri AR, Rizvi MM, Chitnis CE. Development of quantitative receptor-ligand binding assay for use as a tool to estimate immune responses against Plasmodium vivax Duffy binding protein region II. J Immunoassay Immunochem. 2012;33(4):403-413. 